\title{
CO Emission in Infrared-selected Active Galactic Nuclei
}

\author{
Allison Kirkpatrick ${ }^{1,2}$, Chelsea Sharon ${ }^{3,4}$ (10), Erica Keller ${ }^{5,6}$, and Alexandra Pope ${ }^{5}$ (1) \\ ${ }^{1}$ Department of Physics \& Astronomy, University of Kansas, Lawrence, KS 66045, USA; akirkpatrick@ku.edu \\ ${ }^{2}$ Yale Center for Astronomy \& Astrophysics, New Haven, CT 06520, USA \\ ${ }^{3}$ Yale-NUS College, 138527, Singapore \\ ${ }^{4}$ Department of Physics \& Astronomy, McMaster University, Hamilton, Ontario L8S-4M1, Canada \\ ${ }^{5}$ Department of Astronomy, University of Massachusetts, Amherst, MA 01002, USA \\ ${ }^{6}$ National Radio Astronomy Observatory, Charlottesville, VA, 22903, USA \\ Received 2019 January 23; revised 2019 May 9; accepted 2019 May 15; published 2019 July 2
}

\begin{abstract}
In order to better understand how active galactic nuclei (AGNs) affect the interstellar media of their host galaxies, we perform a meta-analysis of the $\mathrm{CO}$ emission for a sample of $z=0.01-4$ galaxies from the literature with existing CO detections and well-constrained AGN contributions to the infrared (67 galaxies). Using either Spitzer/ IRS mid-infrared spectroscopy or Spitzer + Herschel colors we determine the fraction of the infrared luminosity in each galaxy that can be attributed to heating by the AGNs or stars. We calculate new average CO spectral line ratios (primarily from Carilli \& Walter) to uniformly scale the higher-J CO detections to the ground state and accurately determine our sample's molecular gas masses. We do not find significant differences in the gas depletion timescales/star formation efficiencies as a function of the mid-infrared AGN strength $\left(f(\mathrm{AGN})_{\mathrm{MIR}}\right.$ or $\left.L_{\mathrm{IR}}(\mathrm{AGN})\right)$, which indicates that the presence of an infrared-bright AGN is not a sufficient signpost of galaxy quenching. We also find that the dust-to-gas ratio is consistent for all sources, regardless of AGN emission, redshift, or $L_{\mathrm{IR}}$, indicating that dust is likely a reliable tracer of gas mass for massive dusty galaxies (albeit with a large degree of scatter). Finally, if we classify galaxies as either AGN or star formation dominated, we do not find a robust statistically significant difference between their $\mathrm{CO}$ excitation.
\end{abstract}

Key words: galaxies: active - galaxies: evolution - galaxies: high-redshift - galaxies: ISM - quasars: general

\section{Introduction}

The production of luminous quasars is a dramatic story, wherein two immense galaxies collide, fueling a burst of star formation and triggering rapid growth of a supermassive black hole (e.g., Sanders \& Mirabel 1996; Hopkins et al. 2006; Goulding et al. 2018). In this scenario, the active galactic nucleus (AGN) goes through an obscured growth phase, where the accretion disk is hidden by a dust torus. This phase ends when the AGN launches winds powerful enough to blow away some of the circumnuclear obscuring dust so that the accretion disk becomes visible in the optical (Glikman et al. 2012). As the winds clear away the circumnuclear dust, the galaxy's star formation quenches due to galaxy-scale, AGN-driven outflows (Page et al. 2012; Pontzen et al. 2017). However, evidence for this scenario is strongly linked to observational biases. In large statistical samples of optically selected AGNs (Stanley et al. 2017) and X-ray+IR-selected AGNs (Harrison et al. 2012), no correlation between AGN luminosity and decreased star formation rate is observed. On the other hand, theoretical models suggest AGNs may occur at a special phase in a galaxy's life, immediately prior to quenching (Caplar et al. 2018). X-ray-selected AGNs at $z \sim 1-2$ are observed to occur after a galaxy has compactified but before star formation decreases in the center of the galaxy (Kocevski et al. 2017).

At $z>1$, galaxies both on and above the main sequence (e.g., Brinchmann et al. 2004; Daddi et al. 2007; Elbaz et al. 2007; Noeske et al. 2007) appear to have higher gas masses, gas mass fractions, and star formation rates (SFRs) than local galaxies (e.g., Tacconi et al. 2013; Scoville et al. 2016). The high SFRs and gas masses of dusty galaxies are a particular challenge for simulation work, both in terms of reproducing the characteristics of these high- $z$ systems and in terms of the resulting "red and dead" galaxy populations that would exist today (e.g., Casey et al. 2014 and references therein; though see Narayanan et al. 2015). Given the tight correlation observed between galaxies' black hole masses and bulge masses at $z \sim 0$ (implied by the $M_{\mathrm{BH}}-\sigma$ relation; Ferrarese \& Merritt 2000; Gebhardt et al. 2000) and the concurrent peaks of both AGNs and star formation activity at $z \sim 2$, theoretical studies have suggested that AGNs may play a role in quenching the high SFRs of massive galaxies in the early universe (e.g., Somerville et al. 2008).

However, AGNs may not be solely responsible for quenching star formation. Recently, Spilker et al. (2018) found molecular mass outflow rates a factor of two higher than the SFR in a star-forming galaxy at $z=5.3$ that lacked an AGN. In contrast, Hunt et al. (2018) find a quenched galaxy at $z \sim 0.7$ with a large molecular gas reservoir and no indication of gas outflows. Indeed, post-starburst galaxies, which lack AGN signatures, are seen to retain large molecular gas reservoirs (albeit with depleted dense gas; French et al. 2015, 2018; Suess et al. 2017). How these results fit with growing supermassive black holes into a quenching paradigm remains unclear. Both the relative importance of AGN feedback to stellar feedback (e.g., Bouché et al. 2010; Davé et al. 2011; Shetty \& Ostriker 2012; Kim et al. 2013; Lilly et al. 2013) and exact feedback mechanism for AGNs (outflows versus accretion suppression; e.g., Croton et al. 2006; Hopkins et al. 2006; Gabor et al. 2011; Cicone et al. 2014) are still debated. In addition, recent work suggests that AGNs may locally enhance star formation (e.g., Stacey et al. 2010; Ishibashi \& Fabian 2012; Silk 2013; Mahoro et al. 2017). 
While the influence of AGNs on galaxy-wide scales is unclear, AGNs are known to heat their torus and circumnuclear dust to $>1000 \mathrm{~K}$, producing an IR spectral energy density (SED) that emits as a power law in the near- and mid-IR (Elvis et al. 1994). Empirically, dust-enshrouded AGNs are observed to heat circumnuclear dust out to at least $40 \mu \mathrm{m}$, producing a flattening in the far-IR (Mullaney et al. 2011; Kirkpatrick et al. 2012; Sajina et al. 2012; Symeonidis et al. 2016; Ricci et al. 2017). Physical radiative transfer models of the narrow line region (NLR) also predict a modified blackbody peaking at $\lambda \sim 30-100 \mu \mathrm{m}$ from dust clouds commingled with the ionized gas (Groves et al. 2006). AGNs may therefore have a nonnegligible contribution to the IR luminosity $\left(L_{\mathrm{IR}}\right)$ and thus any inferred IR SFRs if the AGN is not properly accounted for. The far-IR influence of AGNs produces an enhanced dust emission with temperatures of $\sim 100 \mathrm{~K}$ (Kirkpatrick et al. 2012). Gas and dust are largely considered to be commingled within the interstellar medium (ISM), at least locally, implying that AGNs could be heating the molecular reservoirs in the centers of galaxies as well, producing a possible feedback mechanism that does not require outflows. However, at $z>1$, gas fractions have dramatically increased, and there has been some suggestion that the gas reservoirs are more spatially extended than the dust (e.g., Hodge et al. 2015, 2016; Tadaki et al. 2017; but see also Hodge et al. 2019).

If AGNs influence the star formation history of galaxies, their effects may be measured in the molecular ISM that fuels star formation. Molecular outflows have been observed in the $\mathrm{CO}$ rotational lines of luminous Type 1 and Type 2 quasistellar objects (QSOs) at low redshift (e.g., Feruglio et al. 2010; Cicone et al. 2014; Fiore et al. 2017), but outflows are not present in all AGN host galaxies. Very high-excitation CO lines $\left(J_{\text {upper }} \gtrsim 10\right)$ have been observed in some AGNs at all redshifts (e.g., Weiß et al. 2007; Ao et al. 2008; HaileyDunsheath et al. 2012; Spinoglio et al. 2012; Rosenberg et al. 2015), but this component likely represents a small fraction of their total molecular gas reservoirs. At high redshift, the $\mathrm{CO}$ spectral line energy distributions (SLEDs) of Type 1 QSOs are known to peak, on average, at higher- $J$ transitions $\left(J_{\text {upper }} \sim 7\right)$ than those of star-forming submillimeter-selected galaxies (SMGs; $J_{\text {upper }} \sim 5$; e.g., Weiss et al. 2007; Carilli \& Walter 2013). However, when high star formation rate densities can explain SLED excitation (e.g., Narayanan \& Krumholz 2014) it is challenging to isolate the potential effects of the AGNs in driving these more moderate excitation $\mathrm{CO}$ rotational lines.

In this paper, we reanalyze data from the literature to examine whether AGNs that are heating circumnuclear dust may also affect the molecular ISM. In our reanalysis, we demonstrate how different measurements of IR luminosity and AGN heating can cause incongruent conclusions about the state of the ISM in AGNs. In Section 2, we discuss our sample selection. In Section 3, we describe how we assess the presence and strength of obscured AGNs, determine $L_{\mathrm{IR}}$ and the inferred SFRs, and use new average SLEDs from the literature to calibrate the gas masses of our sample. In Section 4, we look for evidence of decreased star formation efficiency (SFE) in AGNs and evaluate CO SLEDs for effects from the central AGNs. Finally, in Section 5, we summarize and lay out future directions for uncovering the true effect of an AGN on its host ISM.

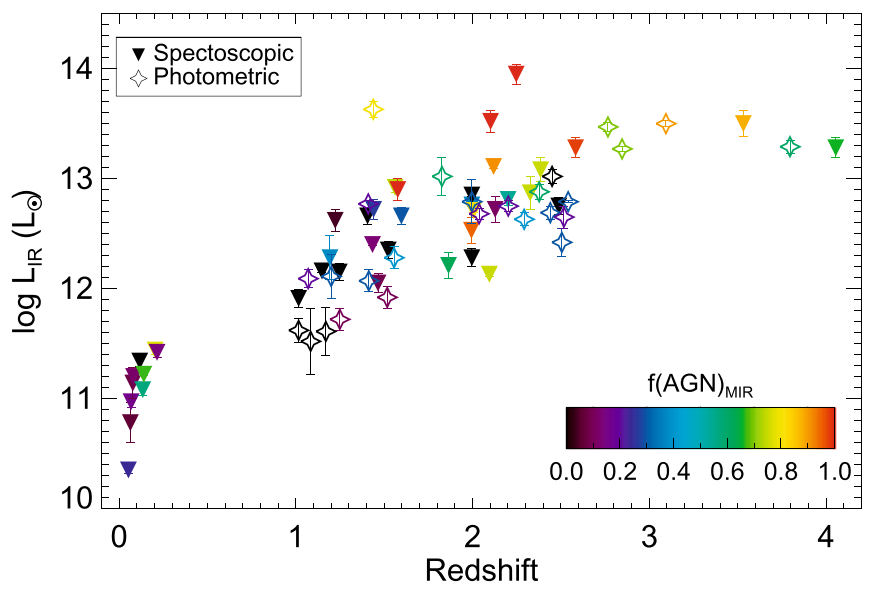

Figure 1. $L_{\mathrm{IR}}-z$ distribution of our sample. The $z<0.5$ sources are from Kirkpatrick et al. (2014), while the $z>1$ galaxies are predominantly mined from the Carilli \& Walter (2013) compilation. We color sources according to their mid-IR AGN contribution. The triangles indicate galaxies where we identified IR AGNs using Spitzer mid-IR spectroscopy, while the stars are galaxies where AGNs were found using IR color diagnostics.

\section{Sample Selection}

We aim to compare the $\mathrm{CO}$ emission properties of IR-selected AGNs with star-forming galaxies (SFGs). Due to the lack of a homogeneous survey, we reanalyze published sources. This technique has also been employed by other authors due to the difficulty of carrying out large-scale molecular gas surveys (e.g., Herrera-Camus et al. 2019; Perna et al. 2018). The meta-analysis necessarily means that our sample will be heterogeneous, although we apply the same analysis techniques uniformly to each source. The relatively recent Carilli \& Walter (2013) annual review described molecular gas in galaxies at $z>1$. This review compiled 173 galaxies with spectroscopic redshifts where molecular gas had been measured, comprising all published measurements at that time. From this parent sample, we initially selected all galaxies with a CO line flux for any $J$ transition that had a magnification $\mu \leqslant 5$, and with at least three photometric data points spanning the range 5-1000 $\mu \mathrm{m}$. We also removed two galaxies (SMM J105131 and SMM J09431+4700) since they are resolved doubles in their $\mathrm{CO}$ emission, but we do not have matching resolved mid-IR colors or IRS spectroscopy to disentangle the AGN emission from the two sources. ${ }^{7}$ We also add in three $70 \mu$ m-selected galaxies (GN70.38, GN70.8, GN70.104) from Pope et al. (2013), where CO was observed with IRAM/PdBI; these galaxies were not in the Carilli \& Walter (2013) compilation. We add in three more QSOs (COB011, no.226, 3C 298) from the Perna et al. (2018) compilation which meet our criteria for full coverage of the IR SED. Additionally, we include 10 galaxies at $z<0.5$ from Kirkpatrick et al. (2014), which have $\mathrm{CO}(1-0)$ measured with the Large Millimeter Telescope, have Herschel observations covering the far-IR, and have Spitzer mid-IR spectroscopy. We have a total heterogenous sample of 67 galaxies. Figure 1 shows the redshift- $L_{\mathrm{IR}}$ distribution of our final sample of 67 galaxies. We list all sources, redshifts, and $f(\mathrm{AGN})_{\mathrm{MIR}}$ in Table 1.

\footnotetext{
SMM J123707+6214 is also a resolved double. However, the combined system has a measured mid-IR AGN fraction of $0 \%$, so we do not have to worry about differences in the two components' AGN fractions. In addition, the $\mathrm{CO}$ line ratios are identical between the two components (Riechers et al. 2011b), so we can treat the system as one for our analysis without any biasing concerns (this effectively under-weights SMM J123707+6214).
} 
Table 1

Sample Positions

\begin{tabular}{|c|c|c|c|c|c|c|}
\hline Name & R.A. & Decl. & $z$ & $f(\mathrm{AGN})_{\mathrm{MIR}^{\mathrm{a}}}{ }^{\mathrm{a}}$ & $\log L_{\mathrm{IR}}\left(L_{\odot}\right)$ & $L_{\mathrm{CO}}^{\prime}\left(\mathrm{K} \mathrm{km} \mathrm{s}^{-1} \mathrm{pc}^{2}\right)$ \\
\hline 5MUSES-179 & 16:08:03.71 & $+54: 53: 02.0$ & 0.053 & $0.24^{\mathrm{a}}$ & $10.35 \pm 0.03$ & $9.16 \pm 0.04$ \\
\hline 5MUSES-169 & 16:04:08.30 & $+54: 58: 13.1$ & 0.064 & $0.06^{\mathrm{a}}$ & $10.78 \pm 0.18$ & $8.92 \pm 0.10$ \\
\hline 5MUSES-105 & $10: 44: 32.94$ & $+56: 40: 41.6$ & 0.068 & $0.16^{\mathrm{a}}$ & $10.97 \pm 0.05$ & $9.19 \pm 0.08$ \\
\hline 5MUSES-171 & $16: 04: 40.64$ & $+55: 34: 09.3$ & 0.078 & $0.08^{\mathrm{a}}$ & $11.14 \pm 0.14$ & $9.33 \pm 0.08$ \\
\hline 5MUSES-229 & $16: 18: 19.31$ & $+54: 18: 59.1$ & 0.082 & $0.12^{\mathrm{a}}$ & $11.20 \pm 0.04$ & $9.55 \pm 0.06$ \\
\hline 5MUSES-132 & $10: 52: 06.56$ & $+58: 09: 47.1$ & 0.117 & $0.00^{\mathrm{a}}$ & $11.34 \pm 0.05$ & $9.44 \pm 0.09$ \\
\hline 5MUSES-227 & $16: 17: 59.22$ & $+54: 15: 01.3$ & 0.134 & $0.57^{\mathrm{a}}$ & $11.08 \pm 0.05$ & $9.00 \pm 0.13$ \\
\hline 5MUSES-141 & $10: 57: 05.43$ & $+58: 04: 37.4$ & 0.140 & $0.67^{\mathrm{a}}$ & $11.22 \pm 0.01$ & $9.46 \pm 0.09$ \\
\hline 5MUSES-136 & $10: 54: 21.65$ & $+58: 23: 44.7$ & 0.204 & $0.77^{\mathrm{a}}$ & $11.44 \pm 0.04$ & $9.67 \pm 0.10$ \\
\hline 5MUSES-216 & $16: 15: 51.45$ & $+54: 15: 36.0$ & 0.215 & $0.14^{\mathrm{a}}$ & $11.42 \pm 0.05$ & $9.62 \pm 0.09$ \\
\hline PEP J123646+621141 & $12: 36: 46.18$ & $+62: 11: 42.0$ & 1.016 & 0.00 & $11.62 \pm 0.11$ & $9.91 \pm 0.15$ \\
\hline GN70.38 & $12: 36: 33.68$ & $+62: 10: 05.9$ & 1.016 & $0.00^{\mathrm{a}}$ & $11.91 \pm 0.08$ & $10.20 \pm 0.16$ \\
\hline EGS 13035123 & $14: 20: 05.40$ & $+53: 01: 15.4$ & 1.070 & 0.20 & $12.09 \pm 0.08$ & $10.39 \pm 0.12$ \\
\hline PEP J123759+621732 & $12: 37: 59.47$ & $+62: 17: 32.9$ & 1.084 & 0.00 & $11.52 \pm 0.30$ & $10.01 \pm 0.16$ \\
\hline GN70.8 & $12: 36: 20.94$ & $+62: 07: 14.2$ & 1.148 & $0.00^{\mathrm{a}}$ & $12.16 \pm 0.01$ & $10.17 \pm 0.15$ \\
\hline PEP J123750+621600 & $12: 37: 50.89$ & $+62: 16: 00.7$ & 1.170 & 0.00 & $11.61 \pm 0.22$ & $10.16 \pm 0.13$ \\
\hline SMM J163658+405728 & $16: 36: 58.78$ & $+40: 57: 27.6$ & 1.193 & $0.38^{\mathrm{a}}$ & $12.28 \pm 0.20$ & $10.35 \pm 0.15$ \\
\hline EGS 13003805 & $14: 19: 40.08$ & $+52: 49: 38.6$ & 1.200 & 0.30 & $12.11 \pm 0.20$ & $10.54 \pm 0.13$ \\
\hline $\mathrm{J} 123634.53+621241.3$ & $12: 36: 34.57$ & $+62: 12: 41.0$ & 1.223 & $0.04^{\mathrm{a}}$ & $12.62 \pm 0.01$ & $10.69 \pm 0.13$ \\
\hline GN70.104 & $12: 37: 02.74$ & $+63: 14: 01.5$ & 1.246 & $0.00^{\mathrm{a}}$ & $12.15 \pm 0.08$ & $10.24 \pm 0.13$ \\
\hline PEP J123712+621753 & $12: 37: 12.15$ & $+62: 17: 54.0$ & 1.249 & 0.10 & $11.72 \pm 0.10$ & $9.66 \pm 0.16$ \\
\hline SMM J030227+000653 & 03:02:27.66 & $+00: 06: 53.0$ & 1.406 & $0.00^{\mathrm{a}}$ & $12.66 \pm 0.08$ & $10.43 \pm 0.13$ \\
\hline EGS 13004291 & 14:19:14.96 & $+52: 49: 30.1$ & 1.410 & 0.20 & $12.77 \pm 0.03$ & $10.73 \pm 0.12$ \\
\hline no. 226 & 03:32:15.99 & $-27: 48: 59.4$ & 1.413 & 0.30 & $12.07 \pm 0.10$ & $10.05 \pm 0.20$ \\
\hline RG J123645.88+620754.2 & $12: 36: 45.88$ & $+62: 07: 54.2$ & 1.434 & $0.13^{\mathrm{a}}$ & $12.40 \pm 0.01$ & $10.41 \pm 0.16$ \\
\hline $3 \mathrm{C} 298$ & 14:19:08.18 & $+06: 28: 34.8$ & 1.438 & 0.80 & $13.63 \pm 0.07$ & $11.63 \pm 0.12$ \\
\hline HR10 & $16: 45: 02.26$ & $+46: 26: 26.5$ & 1.440 & $0.27^{\mathrm{a}}$ & $12.72 \pm 0.09$ & $10.82 \pm 0.07$ \\
\hline BzK-4171 & $12: 36: 26.52$ & $+62: 08: 35.4$ & 1.465 & $0.09^{\mathrm{a}}$ & $12.05 \pm 0.09$ & $10.40 \pm 0.14$ \\
\hline 51613 & $10: 02: 43.36$ & $+01: 34: 20.9$ & 1.517 & 0.10 & $11.92 \pm 0.10$ & $10.38 \pm 0.11$ \\
\hline BzK-21000 & $12: 37: 10.60$ & $+62: 22: 34.6$ & 1.523 & $0.00^{\mathrm{a}}$ & $12.35 \pm 0.08$ & $10.39 \pm 0.11$ \\
\hline 51858 & $10: 02: 40.43$ & $+01: 34: 13.1$ & 1.556 & 0.40 & $12.28 \pm 0.10$ & $10.10 \pm 0.13$ \\
\hline MIPS 8342 & $17: 14: 11.55$ & $+60: 11: 09.3$ & 1.562 & $0.74^{\mathrm{a}, \mathrm{b}}$ & $12.92 \pm 0.05$ & $11.66 \pm 0.09$ \\
\hline $3 \mathrm{C} 318$ & 05:20:05.49 & $+20: 16: 05.7$ & 1.577 & $1.00^{\mathrm{a}}$ & $12.90 \pm 0.10$ & $10.76 \pm 0.14$ \\
\hline SMM J105151+572636 & $10: 51: 51.77$ & $+57: 26: 35.3$ & 1.597 & $0.30^{\mathrm{a}}$ & $12.66 \pm 0.08$ & $10.47 \pm 0.25$ \\
\hline COSB011 & 10:00:38.02 & $+02: 08: 22.9$ & 1.827 & 0.60 & $13.02 \pm 0.17$ & $10.92 \pm 0.14$ \\
\hline SMM J123555+620901 & $12: 35: 54.85$ & $+62: 08: 54.7$ & 1.864 & $0.63^{\mathrm{a}, \mathrm{b}}$ & $12.21 \pm 0.12$ & $10.82 \pm 0.17$ \\
\hline SMM J123632+620800 & $12: 36: 33.04$ & $+62: 08: 05.1$ & 1.994 & $0.95^{\mathrm{a}, \mathrm{b}}$ & $12.53 \pm 0.12$ & $10.81 \pm 0.17$ \\
\hline SMM J123712+621322 & $12: 37: 12.12$ & $+62: 13: 22.2$ & 1.996 & $0.00^{\mathrm{a}}$ & $12.28 \pm 0.08$ & $10.66 \pm 0.18$ \\
\hline SMM J123618.33+621550.5 & $12: 36: 18.47$ & $+62: 15: 51.0$ & 1.996 & $0.36^{\mathrm{a}}$ & $12.76 \pm 0.01$ & $10.70 \pm 0.16$ \\
\hline SMM J123711+621325 & $12: 37: 11.19$ & $+62: 13: 31.2$ & 1.996 & $0.00^{\mathrm{a}}$ & $12.85 \pm 0.01$ & $10.86 \pm 0.16$ \\
\hline RG J123711 & $12: 37: 11.34$ & $+62: 32: 31.0$ & 1.996 & 0.30 & $12.79 \pm 0.20$ & $10.43 \pm 0.18$ \\
\hline MIPS 16080 & $17: 18: 44.77$ & $+60: 01: 15.9$ & 2.006 & $0.81^{\mathrm{a}, \mathrm{b}}$ & $12.71 \pm 0.02$ & $10.51 \pm 0.14$ \\
\hline SMM J021738-050339 & $02: 17: 38.68$ & $-05: 03: 39.3$ & 2.037 & 0.20 & $12.68 \pm 0.05$ & $10.94 \pm 0.16$ \\
\hline RG J123644.13+621450.7 & $12: 36: 44.13$ & $+62: 14: 50.7$ & 2.095 & $0.75^{\mathrm{a}}$ & $12.13 \pm 0.02$ & $10.52 \pm 0.17$ \\
\hline HS $1002+4400$ & 10:05:17.43 & $+43: 46: 09.3$ & 2.102 & $1.00^{\mathrm{a}}$ & $13.52 \pm 0.10$ & $11.07 \pm 0.12$ \\
\hline MIPS 15949 & $17: 21: 09.22$ & $+60: 15: 01.3$ & 2.119 & $0.91^{\mathrm{a}, \mathrm{b}}$ & $13.11 \pm 0.02$ & $10.66 \pm 0.12$ \\
\hline MIPS 16144 & $17: 24: 22.09$ & $+59: 31: 50.8$ & 2.128 & $0.11^{\mathrm{a}}$ & $12.72 \pm 0.12$ & $10.79 \pm 0.14$ \\
\hline MIPS 429 & $17: 16: 11.83$ & $+59: 12: 13.2$ & 2.201 & $0.54^{\mathrm{a}}$ & $12.81 \pm 0.05$ & $10.66 \pm 0.14$ \\
\hline SMM J123549+6215 & $12: 35: 49.43$ & $+62: 15: 36.7$ & 2.202 & 0.20 & $12.75 \pm 0.05$ & $10.88 \pm 0.05$ \\
\hline RX J124913.86-055906.2 & $12: 49: 13.85$ & $-05: 59: 19.4$ & 2.247 & $1.00^{\mathrm{a}}$ & $13.95 \pm 0.09$ & $10.72 \pm 0.10$ \\
\hline SMM J021725-045934 & $02: 17: 25.16$ & $-04: 59: 34.9$ & 2.292 & 0.40 & $12.63 \pm 0.06$ & $10.68 \pm 0.16$ \\
\hline MIPS 16059 & $17: 24: 28.45$ & $+60: 15: 33.0$ & 2.326 & $0.75^{\mathrm{a}, \mathrm{b}}$ & $12.87 \pm 0.15$ & $10.45 \pm 0.14$ \\
\hline SMM J16371+4053 & $16: 37: 06.50$ & $+40: 53: 14.0$ & 2.377 & $0.60^{\mathrm{b}}$ & $12.88 \pm 0.07$ & $10.53 \pm 0.15$ \\
\hline SMM J163650+405734 & $16: 36: 50.41$ & $+40: 57: 34.4$ & 2.385 & $0.75^{\mathrm{a}, \mathrm{b}}$ & $13.08 \pm 0.11$ & $11.01 \pm 0.13$ \\
\hline SMM J105227+572512 & $10: 52: 27.10$ & $+57: 25: 16.0$ & 2.440 & 0.30 & $12.69 \pm 0.06$ & $10.38 \pm 0.16$ \\
\hline SMM J16358+4105 & $16: 36: 58.20$ & $+41: 05: 24.0$ & 2.450 & 0.00 & $13.02 \pm 0.06$ & $11.03 \pm 0.08$ \\
\hline SMM J123707+6214 & $12: 37: 07.28$ & $+62: 14: 08.6$ & 2.488 & $0.00^{\mathrm{a}}$ & $12.75 \pm 0.08$ & $10.99 \pm 0.05$ \\
\hline SMM J123606+621047 & $12: 36: 06.85$ & $+62: 10: 21.4$ & 2.505 & 0.30 & $12.42 \pm 0.13$ & $10.41 \pm 0.18$ \\
\hline SMM J221804+002154 & 22:18:04.42 & $+00: 21: 54.4$ & 2.517 & 0.20 & $12.65 \pm 0.10$ & $10.71 \pm 0.15$ \\
\hline SMM J021738-050528 & $02: 17: 38.88$ & $-05: 05: 28.2$ & 2.541 & 0.30 & $12.79 \pm 0.01$ & $10.99 \pm 0.15$ \\
\hline VCV J1409+5628 & 14:09:55.50 & $+56: 28: 27.0$ & 2.583 & $0.98^{\mathrm{a}}$ & $13.28 \pm 0.09$ & $10.93 \pm 0.11$ \\
\hline AMS12 & $17: 18: 22.65$ & $+59: 01: 54.3$ & 2.766 & 0.70 & $13.47 \pm 0.04$ & $10.87 \pm 0.13$ \\
\hline SMM J04135+10277 & $04: 13: 27.28$ & $+10: 27: 40.4$ & 2.846 & $0.70^{\mathrm{b}}$ & $13.27 \pm 0.02$ & $10.94 \pm 0.08$ \\
\hline B3 J2330+3927 & $23: 30: 24.84$ & $+39: 37: 12.2$ & 3.094 & 0.90 & $13.50 \pm 0.03$ & $11.38 \pm 0.16$ \\
\hline
\end{tabular}


Table 1

(Continued)

\begin{tabular}{|c|c|c|c|c|c|c|}
\hline Name & R.A. & Decl. & $z$ & $f(\mathrm{AGN})_{\mathrm{MIR}^{\mathrm{a}}}$ & $\log L_{\mathrm{IR}}\left(L_{\odot}\right)$ & $L_{\mathrm{CO}}^{\prime}\left(\mathrm{K} \mathrm{km} \mathrm{s}^{-1} \mathrm{pc}^{2}\right)$ \\
\hline $6 \mathrm{C} 1909+722$ & $19: 08: 23.70$ & $+72: 20: 11.6$ & 3.532 & $0.88^{\mathrm{a}}$ & $13.50 \pm 0.12$ & $11.07 \pm 0.10$ \\
\hline $4 \mathrm{C} 41.17$ & $06: 50: 52.10$ & $+41: 30: 30.5$ & 3.796 & 0.60 & $13.29 \pm 0.06$ & $11.26 \pm 0.15$ \\
\hline GN20 & $12: 37: 11.86$ & $+62: 22: 12.6$ & 4.055 & $0.66^{\mathrm{a}}$ & $13.28 \pm 0.09$ & $11.15 \pm 0.10$ \\
\hline
\end{tabular}

Notes.

a These sources had Spitzer IRS spectroscopy which was decomposed to measure the mid-IR AGN fraction.

${ }^{\mathrm{b}}$ We identified these sources as IR AGNs, but they are classified as SFGs in Carilli \& Walter (2013).

\section{Methods: AGN Fraction, SFR, $M_{\text {dust }}$, and $L_{\mathrm{CO}}^{\prime}$}

\subsection{AGN Fractions from Spitzer Spectroscopy and IR Colors}

We use either Spitzer IRS spectroscopy (available for 41 galaxies) or IR color diagnostics (26 galaxies) to locate AGNs within our sample. We quantify $f(\mathrm{AGN})_{\mathrm{MIR}}$, the fraction of mid-IR $(5-15 \mu \mathrm{m})$ luminosity arising from an AGN torus. We do not perform a full SED decomposition into multiple components as has become common in the literature because this risks overfitting and overinterpreting the sparse amount of IR data available.

The low-resolution $(R=\lambda / \Delta \lambda \sim 100)$ Spitzer IRS spectra were reduced following the method outlined in Pope et al. (2008). We used the Spitzer IRS spectra to calculate the AGN contribution to the mid-IR luminosity following the technique described in Pope et al. (2008) and Kirkpatrick et al. (2015). We fit the individual spectra with a model comprised of three components: (1) the star formation component is represented by the mid-IR spectrum of the prototypical starburst M82; (2) the AGN component is determined by fitting a pure power law with the slope and normalization as free parameters; (3) an extinction curve from the Draine (2003) dust models is applied to the AGN component; for our wavelength range, the extinction curve primarily constrains the $9.7 \mu \mathrm{m}$ silicate absorption feature. We fit all three components simultaneously. We integrate under the best-fit model and the power-law component to measure $f(\mathrm{AGN})_{\mathrm{MIR}}$. Our spectroscopic measurement technique is uncertain by less than $10 \%$ in $f(\mathrm{AGN})_{\mathrm{MIR}}$ (Kirkpatrick et al. 2015).

For the 26 sources that lack mid-IR spectroscopy, we determine $f(\mathrm{AGN})_{\mathrm{MIR}}$ using the Spitzer + Herschel color classification as described in Kirkpatrick et al. (2017), which is based on observed AGN emission in galaxies at $z \sim 1-3$. We create a redshift-dependent diagnostic that assigns an $f(\mathrm{AGN})_{\mathrm{MIR}}$ depending on the ratio of $S_{8} / S_{3.6}$, which at $z>1$ separates AGNs on the basis of a hot torus as compared with the $1.6 \mu \mathrm{m}$ stellar bump seen in SFGs. This is combined with either $S_{250} / S_{24}$ or $S_{100} / S_{24}$, which trace the relative amounts of cold and hot dust (since AGN should have a larger hot dust component). Color determination of $f(\mathrm{AGN})_{\mathrm{MIR}}$ in this manner is accurate to $30 \%$ (Kirkpatrick et al. 2017).

Our mid-IR classifications largely agree with those of Carilli $\&$ Walter (2013). That is, sources with $f(\mathrm{AGN})_{\text {MIR }}<0.5$ are predominately listed as a type of star-forming galaxy in Carilli \& Walter, and the radio AGNs and QSOs identified in Carilli \& Walter all have $f(\mathrm{AGN})_{\mathrm{MIR}} \geqslant 0.6$. In addition, we identify nine additional galaxies as AGNs that were originally listed as SFGs by Carilli \& Walter. We note our 9 "new" AGN in Table 1.

\section{2. $\mathrm{L}_{I R}$ and $S F R S$}

We gathered all available photometry for our sample using the Spitzer/IRSA and NED databases. The number of far-IR data points per source varies. To allow the greatest flexibility when determining $L_{\mathrm{IR}}$, we combine a template from Kirkpatrick et al. (2015), which is empirically derived from galaxies at $z \sim 1-3$, with a far-IR model consisting of two modified blackbodies. The number of data points at $\lambda>20 \mu \mathrm{m}$ determines how many far-IR parameters we allow to vary, so that we are at most fitting for $n-1$ parameters. At a minimum, we only fit for the normalization of the cold component, and at a maximum, we fit both normalizations and the cold dust temperature. The warm dust temperature is held fixed at 65-90 K, where using a warmer dust temperature depends on the presence of an AGN (Kirkpatrick et al. 2012). Here, we use a warmer dust temperature when $f(\mathrm{AGN})_{\mathrm{MIR}}>0.6$, which is when this component starts to contribute measurably $(>20 \%)$ to the far-IR SED (Kirkpatrick et al. 2015). We choose this simple method rather than using a library of models because we do not want to risk overfitting a scarcity of data points in many sources. As we are not trying to measure dust temperatures or $\beta$, we are not concerned with degeneracies between the parameters. $L_{\mathrm{IR}}$ is robust against small variations in $T$ and $\beta$ as long as the model fits all the data points. The Kirkpatrick et al. (2015) library provides templates based on $f(\mathrm{AGN})_{\mathrm{MIR}}$, and we use the appropriate template for each source to complete the best-fit model at $\lambda \lesssim 20 \mu \mathrm{m}$. In Figure 2 we illustrate how our technique of modifying templates is more accurate than simply scaling a template to a photometric data point.

The $L_{\mathrm{IR}}$ can be converted to an SFR once the AGN heating is removed. Due to the disparate data available for each source, we are unable to decompose the full SEDs into an AGN and star-forming component. However Kirkpatrick et al. (2015) demonstrate that $f(\mathrm{AGN})_{\mathrm{MIR}}$ is related to $f(\mathrm{AGN})_{\mathrm{IR}}$, the AGN contribution to $L_{\mathrm{IR}}(8-1000 \mu \mathrm{m})$, through the quadratic relation

$$
f(\mathrm{AGN})_{\mathrm{IR}}=0.66 \times f(\mathrm{AGN})_{\mathrm{MIR}}^{2}-0.035 \times f(\mathrm{AGN})_{\mathrm{MIR}} .
$$

We correct $L_{\mathrm{IR}}$ for AGN heating using $f(\mathrm{AGN})_{\mathrm{IR}}$ using

$$
L_{\mathrm{IR}}^{\mathrm{SF}}=L_{\mathrm{IR}} \times\left(1-f(\mathrm{AGN})_{\mathrm{IR}}\right)
$$

and then convert $L_{\mathrm{IR}}^{\mathrm{SF}}$ to a SFR following Murphy et al. (2011):

$$
\mathrm{SFR}\left[M_{\odot} \mathrm{yr}^{-1}\right]=\left(1.59 \times 10^{-10}\right) \times L_{\mathrm{IR}}^{\mathrm{SF}}\left[L_{\odot}\right] .
$$

\subsection{Dust Masses}

In order to explore whether increased AGN heating of the ISM affects the gas-to-dust ratio in galaxies, we use two methods for determining the dust mass in our sample. 


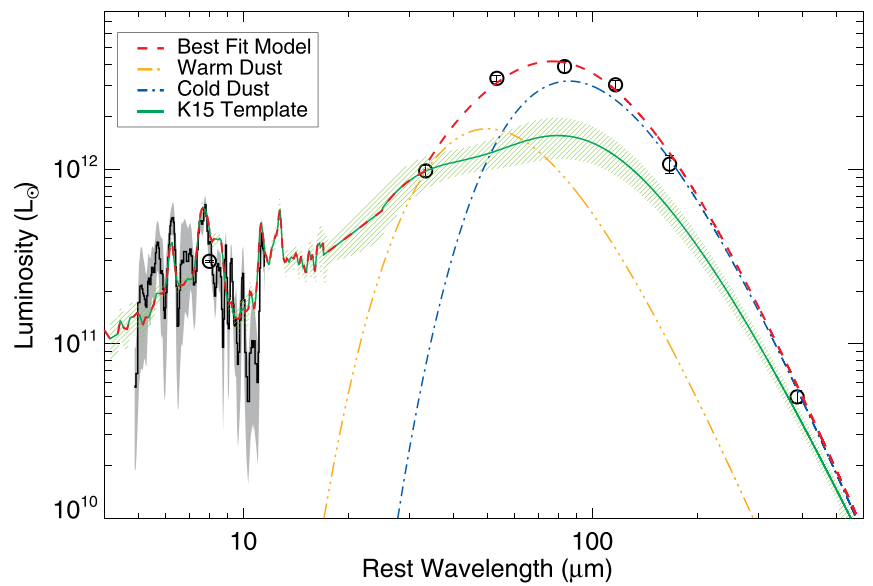

Figure 2. Example showing our technique of calculating $L_{\mathrm{IR}}$ by combining a template (green solid line) from Kirkpatrick et al. (2015) with a twotemperature modified blackbody model in the far-IR. The warm component is represented by the orange dotted-dashed line and cold component by the blue dotted-dashed line. The combined best-fit model is shown by the red dashed line. This method more accurately determines $L_{\mathrm{IR}}$ than the template on its own.

For the first method, we calculate the dust mass and characteristic radius of the dust emission using the physically motivated, self-consistent radiative transfer model from Chakrabarti \& McKee $(2005,2008)$. The far-IR SED can be characterized by the ratio of $L_{\mathrm{IR}}$ to $M_{\text {dust }}$ and the radius of the source for a given dust opacity (Witt \& Gordon 2000; Misselt et al. 2001; Chakrabarti \& McKee 2005, 2008). The authors use the Weingartner \& Draine (2001) dust models with $R_{V}=5.5$ to parameterize the grain composition and emission properties. It should be noted that the choice of $R_{V}$ has a negligible effect in the far-IR, where $M_{\text {dust }}$ is calculated. The authors make the simplifying assumption that star-forming regions can be represented as homogeneous and spherically symmetric. Each star-forming region is surrounded by spherical shells of dust, and the temperature and density profiles of the dust shells are approximated as power laws. The radiative transfer code also calculates a far-IR luminosity $(\lambda>30 \mu \mathrm{m})$, which is consistent with our $L_{\mathrm{IR}}$ calculated above. Many of our galaxies lack enough data to fit the near-, mid-, and far-IR with a robust dust model (such as Draine \& Li 2007) that calculates grain populations. As the relative abundance of small grains and carbonaceous grains does not have a significant effect on the large-grain submillimeter emission, we opt for a model that only fits the far-IR. We list $M_{\text {dust }}^{\mathrm{CM}}$ and $R$ from the Chakrabarti \& McKee (2005) model in Table 2.

For comparison to the Chakrabarti \& McKee (2005) model, we also calculate the dust mass using the simple model of Scoville et al. (2016). In this model, the dust mass is calculated using any submillimeter observation with $\lambda_{\text {rest }}>250 \mu \mathrm{m}$, and assuming a standard cold dust temperature of $25 \mathrm{~K}$. Several of our galaxies lack a submillimeter observation at $\lambda_{\text {rest }}>250 \mu \mathrm{m}$, so we are unable to calculate a dust mass in this manner. We compare these dust masses, $M_{\text {dust }}^{\mathrm{S}}$ and $M_{\text {dust }}^{\mathrm{CM}}$, in Figure 3 ; their values are broadly consistent. The large errors on many $M_{\text {dust }}^{\mathrm{CM}}$ indicate the unreliability of fitting a multi-component model to a scarce number of data points. Some of our galaxies only had two photometric measurements beyond $\lambda=30 \mu \mathrm{m}$.

\section{4. $L_{\mathrm{CO}}^{\prime}$ and Gas Masses}

3.4.1. Excitation Correction

Our $f(\mathrm{AGN})_{\mathrm{MIR}}$ sample has been observed in many different $\mathrm{CO}$ lines. In order to determine $L_{\mathrm{CO}}^{\prime}$ and the associated gas masses free from excitation effects, assumptions need to be made about the line ratios in objects that do not have measurements of the $\mathrm{CO}(1-0)$ line (the ground-state rotational transition that best traces the total molecular gas). Bothwell et al. (2013) produced an average CO SLED for a sample of $z \sim 1-4$ SMGs with spectroscopic redshifts (generally from Ly $\alpha, \mathrm{H} \alpha$, or polycyclic aromatic hydrocarbon observations). However, there are significantly more $\mathrm{CO}$ detections currently in the literature, particularly more objects with $\mathrm{CO}(1-0)$ detections and more objects at higher redshifts.

We therefore construct a new average CO SLED using the entire library of high- $z$ CO detections from Carilli \& Walter (2013), supplemented with new CO detections from Pope et al. (2013), Aravena et al. (2014), Sharon et al. (2016), Yang et al. (2017), Frayer et al. (2018), Perna et al. (2018), and references therein. We have included the entire library, as opposed to just those sources with well-sampled IR SEDs, in order to make use of all the available molecular gas information and therefore reduce uncertainties on our new SLED.

To construct the average CO SLED, we follow Bothwell et al. (2013). We linearly scale all sources' line fluxes and uncertainties by the ratio of their far-IR luminosity to the approximate median far-IR luminosity of the full sample $\left(5 \times 10^{11} L_{\odot}\right)$ in order to prevent the brightest sources from biasing later averages. For each $\mathrm{CO}$ transition, we find the median scaled line luminosity for all sources detected in that line. We determine the uncertainty on the median scaled line luminosity via bootstrapping (with replacement) over 10,000 iterations, perturbing the random subset of line fluxes by their uncertainties in each iteration. We note that many sources from Carilli \& Walter (2013) have small uncertainties. We assume that if those uncertainties are less than $10 \%$ of the measured value, then only the statistical uncertainty is reported. For those objects, we add an additional $10 \%$ uncertainty in quadrature to the reported value in order to approximate flux calibration uncertainties. For the very few line measurements with missing uncertainties, we assume a $20 \%$ uncertainty. Note that this analysis only includes reported line detections, which may produce a median SLED biased both toward the characteristics of the brighter classes of objects that are more commonly observed (which cannot be accounted for by luminosity scaling) and toward higher-excitation specifically (since upper limits from higher- $J$ non-detections are not included in the analysis).

We give the ratio of the median line luminosity to the median $\mathrm{CO}(1-0)$ line luminosity (using the scaled measured values) and the standard deviation of the ratio (from bootstrapping) in Table 3. The line ratios (in units of brightness temperature) can be related to the line luminosity and integrated line flux by

$$
r_{J, 1}=\frac{L_{\mathrm{CO}(J \rightarrow J-1)}^{\prime}}{L_{\mathrm{CO}(1 \rightarrow 0)}^{\prime}}=\frac{S \Delta v_{\mathrm{CO}(J \rightarrow J-1)}}{S \Delta v_{\mathrm{CO}(1 \rightarrow 0)}}\left(\frac{1}{J}\right)^{2} .
$$

In Figure 4, we plot the median CO SLED, as well as the individual line ratios contributing to that SLED. For individual 
Table 2

Sample Derived Gas and Dust Properties

\begin{tabular}{|c|c|c|c|c|c|c|}
\hline Name & $\alpha_{\mathrm{CO}}{ }^{\mathrm{a}}$ & $R(\mathrm{kpc})^{\mathrm{b}}$ & $\log M_{\text {dust }}^{\mathrm{CM}}\left(M_{\odot}\right)^{\mathrm{b}}$ & $\log M_{\text {dust }}^{\mathrm{S}}\left(M_{\odot}\right)^{\mathrm{c}}$ & $\log M_{\text {gas }}^{\mathrm{V}}\left(M_{\odot}\right)^{\mathrm{d}}$ & $\log M_{\mathrm{gas}}^{\mathrm{MS}}\left(M_{\odot}\right)^{\mathrm{e}}$ \\
\hline 5MUSES-179 & 0.45 & 0.11 & $7.23 \pm 0.10$ & $7.11 \pm 0.04$ & 8.81 & 9.82 \\
\hline 5MUSES-169 & 0.42 & 0.10 & $7.55 \pm 0.25$ & $\ldots$ & 8.55 & 9.58 \\
\hline 5MUSES-105 & 0.26 & 0.06 & $7.49 \pm 0.63$ & $7.39 \pm 0.09$ & 8.60 & 9.85 \\
\hline 5MUSES-171 & 0.39 & 0.19 & $7.86 \pm 0.13$ & $\ldots$ & 8.92 & 9.99 \\
\hline 5MUSES-229 & 0.21 & 0.09 & $7.86 \pm 0.66$ & $7.74 \pm 0.03$ & 8.87 & 10.21 \\
\hline 5MUSES-132 & 0.41 & 0.22 & $7.82 \pm 0.09$ & $7.83 \pm 0.03$ & 9.05 & 10.10 \\
\hline 5MUSES-227 & 0.24 & 0.02 & $6.65 \pm 0.77$ & $7.15 \pm 0.08$ & 8.38 & 9.66 \\
\hline 5MUSES-141 & 0.30 & 0.14 & $7.85 \pm 0.23$ & $7.65 \pm 0.04$ & 8.93 & 10.12 \\
\hline 5MUSES-136 & 0.29 & 0.15 & $7.67 \pm 0.14$ & $7.82 \pm 0.04$ & 9.14 & 10.33 \\
\hline 5MUSES-216 & 0.30 & 0.19 & $7.97 \pm 0.17$ & $7.89 \pm 0.02$ & 9.09 & 10.28 \\
\hline PEP J123646+621141 & 0.28 & 0.34 & $8.33 \pm 0.12$ & $\ldots$ & 9.36 & 10.57 \\
\hline GN70.38 & 0.22 & 0.35 & $8.38 \pm 0.13$ & $8.27 \pm 0.49$ & 9.56 & 10.86 \\
\hline EGS 13035123 & 0.22 & 0.67 & $8.94 \pm 0.13$ & $\cdots$ & 9.73 & 11.05 \\
\hline PEP J123759+621732 & 0.25 & 0.36 & $8.49 \pm 0.16$ & $\cdots$ & 9.41 & 10.67 \\
\hline GN70.8 & 0.24 & 0.36 & $8.34 \pm 0.11$ & $8.64 \pm 0.24$ & 9.55 & 10.83 \\
\hline PEP J123750+621600 & 0.24 & 0.33 & $8.24 \pm 0.11$ & $\ldots$ & 9.55 & 10.82 \\
\hline SMM J163658+405728 & 0.25 & 0.74 & $8.85 \pm 0.09$ & $8.89 \pm 0.12$ & 9.75 & 11.01 \\
\hline EGS 13003805 & 0.21 & 0.43 & $8.33 \pm 0.08$ & $\cdots$ & 9.86 & 11.20 \\
\hline $\mathrm{J} 123634.53+621241.3$ & 0.20 & 0.67 & $8.72 \pm 0.08$ & $8.46 \pm 0.36$ & 9.98 & 11.35 \\
\hline GN70.104 & 0.25 & 0.42 & $8.38 \pm 0.09$ & $8.74 \pm 0.17$ & 9.63 & 10.90 \\
\hline PEP J123712+621753 & 0.35 & 0.19 & $7.63 \pm 0.08$ & $\cdots$ & 9.21 & 10.32 \\
\hline SMM J030227+000653 & 0.24 & 0.64 & $8.63 \pm 0.07$ & $8.84 \pm 0.13$ & 9.82 & 11.09 \\
\hline EGS 13004291 & 0.18 & 0.57 & $8.63 \pm 0.09$ & $\ldots$ & 9.99 & 11.39 \\
\hline no. 226 & 0.24 & 0.26 & $8.12 \pm 0.13$ & $8.84 \pm 0.13$ & 9.44 & 10.71 \\
\hline RG J123645.88+620754.2 & 0.24 & 0.42 & $8.24 \pm 0.07$ & $7.88 \pm 1.38$ & 9.78 & 11.07 \\
\hline $3 \mathrm{C} 298$ & 0.23 & 8.22 & $10.07 \pm 0.01$ & $9.51 \pm 0.04$ & 10.98 & 12.29 \\
\hline HR10 & 0.10 & 0.31 & $8.96 \pm 0.63$ & $9.15 \pm 0.13$ & 9.80 & 11.48 \\
\hline BzK-4171 & 0.21 & 0.34 & $8.20 \pm 0.09$ & $7.86 \pm 1.35$ & 9.73 & 11.06 \\
\hline 51613 & 0.20 & 0.75 & $9.24 \pm 0.20$ & $\ldots$ & 9.68 & 11.04 \\
\hline BzK-21000 & 0.10 & 0.08 & $7.95 \pm 0.84$ & $8.46 \pm 0.24$ & 9.38 & 11.05 \\
\hline 51858 & 0.26 & 0.55 & $8.80 \pm 0.14$ & $\cdots$ & 9.51 & 10.76 \\
\hline MIPS 8342 & 0.10 & 0.44 & $8.16 \pm 0.05$ & $8.65 \pm 0.23$ & 10.67 & 12.32 \\
\hline $3 \mathrm{C} 318$ & 0.17 & 0.60 & $8.75 \pm 0.10$ & $9.47 \pm 0.11$ & 9.99 & 11.42 \\
\hline SMM J105151+572636 & 0.20 & 0.57 & $8.78 \pm 0.12$ & $8.85 \pm 0.16$ & 9.78 & 11.13 \\
\hline COSB011 & 0.18 & 0.75 & $8.69 \pm 0.06$ & $\cdots$ & 10.18 & 11.58 \\
\hline SMM J123555+620901 & 0.15 & 0.51 & $8.70 \pm 0.13$ & $8.92 \pm 0.15$ & 10.00 & 11.48 \\
\hline SMM J123632+620800 & 0.08 & 0.15 & $8.54 \pm 1.05$ & $8.92 \pm 0.10$ & 9.70 & 11.47 \\
\hline SMM J123712+621322 & 0.22 & 0.74 & $8.67 \pm 0.06$ & $9.07 \pm 0.07$ & 10.00 & 11.32 \\
\hline SMM J123618.33+621550.5 & 0.23 & 0.94 & $8.84 \pm 0.05$ & $8.88 \pm 0.13$ & 10.06 & 11.36 \\
\hline SMM J123711+621325 & 0.20 & 0.78 & $8.64 \pm 0.05$ & $9.20 \pm 0.06$ & 10.16 & 11.52 \\
\hline RG J123711 & 0.30 & 1.07 & $8.82 \pm 0.04$ & $\cdots$ & 9.91 & 11.09 \\
\hline MIPS 16080 & 0.22 & 0.56 & $8.53 \pm 0.07$ & $8.48 \pm 0.34$ & 9.86 & 11.17 \\
\hline SMM J021738-050339 & 0.17 & 0.74 & $8.79 \pm 0.08$ & $8.84 \pm 0.17$ & 10.17 & 11.60 \\
\hline RG J123644.13+621450.7 & 0.19 & 0.35 & $8.32 \pm 0.12$ & $8.94 \pm 0.09$ & 9.79 & 11.18 \\
\hline HS1002+4400 & 0.38 & 5.99 & $9.55 \pm 0.01$ & $9.26 \pm 0.08$ & 10.64 & 11.73 \\
\hline MIPS 15949 & 0.09 & 0.16 & $8.47 \pm 0.76$ & $8.73 \pm 0.18$ & 9.63 & 11.32 \\
\hline MIPS 16144 & 0.19 & 1.11 & $9.21 \pm 0.09$ & $\cdots$ & 10.07 & 11.45 \\
\hline MIPS 429 & 0.15 & 0.35 & $8.49 \pm 0.17$ & $8.63 \pm 0.24$ & 9.84 & 11.32 \\
\hline SMM J123549+6215 & 0.08 & 0.28 & $9.03 \pm 0.91$ & $9.04 \pm 0.10$ & 9.79 & 11.54 \\
\hline RX J124913.86-055906.2 & 0.41 & 5.99 & $9.83 \pm 0.01$ & $9.04 \pm 0.08$ & 10.34 & 11.38 \\
\hline SMM J021725-045934 & 0.20 & 0.72 & $8.82 \pm 0.08$ & $8.82 \pm 0.18$ & 9.97 & 11.34 \\
\hline MIPS 16059 & 0.18 & 0.33 & $8.40 \pm 0.16$ & $\cdots$ & 9.70 & 11.11 \\
\hline SMM J16371+4053 & 0.37 & 2.24 & $9.16 \pm 0.02$ & $9.21 \pm 0.10$ & 10.10 & 11.19 \\
\hline SMM J163650+405734 & 0.20 & 1.04 & $8.80 \pm 0.04$ & $9.12 \pm 0.08$ & 10.30 & 11.67 \\
\hline SMM J105227+572512 & 0.25 & 0.72 & $8.76 \pm 0.08$ & $8.82 \pm 0.39$ & 9.78 & 11.04 \\
\hline SMM J16358+4105 & 0.10 & 0.28 & $8.53 \pm 0.29$ & $9.20 \pm 0.08$ & 10.02 & 11.69 \\
\hline SMM J123707+6214 & 0.17 & 0.69 & $8.64 \pm 0.06$ & $8.85 \pm 0.14$ & 10.22 & 11.65 \\
\hline SMM J123606+621047 & 0.20 & 0.43 & $8.55 \pm 0.13$ & $\cdots$ & 9.71 & 11.07 \\
\hline SMM J221804+002154 & 0.42 & 5.99 & $9.83 \pm 0.01$ & $9.12 \pm 0.11$ & 10.33 & 11.37 \\
\hline SMM J021738-050528 & 0.18 & 0.83 & $8.77 \pm 0.06$ & $9.02 \pm 0.09$ & 10.24 & 11.65 \\
\hline VCV J1409+5628 & 0.48 & 8.35 & $9.67 \pm 0.01$ & $9.61 \pm 0.02$ & 10.61 & 11.59 \\
\hline AMS12 & 0.73 & 7.68 & $8.93 \pm 0.01$ & $9.15 \pm 0.07$ & 10.73 & 11.53 \\
\hline SMM J04135+10277 & 0.50 & 11.57 & $9.95 \pm 0.01$ & $9.56 \pm 0.05$ & 10.64 & 11.60 \\
\hline B3 J2330+3927 & 0.08 & 0.33 & $8.59 \pm 0.23$ & $9.33 \pm 0.05$ & 10.29 & 12.04 \\
\hline
\end{tabular}


Table 2

(Continued)

\begin{tabular}{lcccccc}
\hline \hline Name & $\alpha_{\mathrm{CO}}{ }^{\mathrm{a}}$ & $R(\mathrm{kpc})^{\mathrm{b}}$ & $\log M_{\text {dust }}^{\mathrm{CM}}\left(M_{\odot}\right)^{\mathrm{b}}$ & $\log M_{\text {dust }}^{\mathrm{S}}\left(M_{\odot}\right)^{\mathrm{c}}$ & $\log M_{\mathrm{gas}}^{\mathrm{V}}\left(M_{\odot}\right)^{\mathrm{d}}$ & 10.46 \\
\hline 6C1909+722 & 0.25 & 1.76 & $8.92 \pm 0.02$ & $\ldots$ & 11.73 & 1.15 \\
4C41.17 & 0.08 & 0.25 & $8.50 \pm 0.34$ & $9.15 \pm 0.04$ & 1.92 & 10.72 \\
GN20 & 0.37 & 6.39 & $9.54 \pm 0.01$ & $9.22 \pm 0.07$ & 11.81 \\
\hline
\end{tabular}

Notes.

${ }^{\text {a }}$ Variable $\alpha_{\mathrm{CO}}$ calculated from Narayanan et al. (2012).

${ }^{\mathrm{b}}$ ISM radius, $R$, and $M_{\text {dust }}^{\mathrm{CM}}$ are the parameters derived by fitting a radiative transfer model to far-IR photometry using the method of Chakrabarti \& McKee (2008).

${ }^{c} M_{\text {dust }}^{\text {S }}$ is calculated using the formalism of Scoville et al. (2016).

${ }^{\mathrm{d}} M_{\mathrm{gas}}^{\mathrm{V}}$ is calculated using the variable $\alpha_{\mathrm{CO}}$ in Column 2. Errors are the same as for $L_{\mathrm{CO}}^{\prime}$ in Table 1.

${ }^{\text {e }} M_{\mathrm{gas}}^{\mathrm{MS}}$ is calculated using a single $\alpha_{\mathrm{CO}}=4.6$ for every galaxy. Errors are the same as for $L_{\mathrm{CO}}^{\prime}$ in Table 1 .

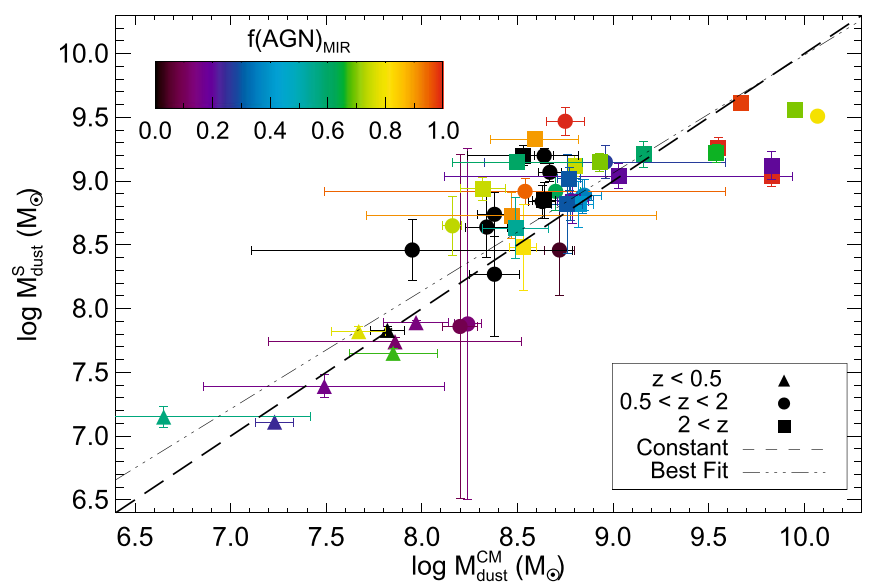

Figure 3. Comparison of dust mass derived from fitting the Chakrabarti \& McKee (2005) models on the $x$-axis with dust mass derived using the method in Scoville et al. (2016) on the $y$-axis. The dashed line is the one-to-one correlation, while the dotted-dashed line shows the best fit to the data using an ordinary least-squares bisector method.

sources that lack $\mathrm{CO}(1-0)$ measurements, we use the median $\mathrm{CO}(1-0)$ flux from our bootstrapped analysis, scaled by the ratio of that source's far-IR luminosity to the fiducial value, in order to calculate and plot an illustrative line ratio for that object.

Many high- $z$ sources studied in $\mathrm{CO}$ are gravitationally lensed due to the advantageous boosting of the observed flux. However, spatially varying magnification factors may interact with spatially varying properties of the galaxy, biasing unresolved measurements of lensed galaxies' fluxes (e.g., Blain 1999; Hezaveh et al. 2012; Serjeant 2012). In a series of simulations, Serjeant (2012) showed that differential lensing tends to scatter unresolved measurements of galaxies' average properties toward those of their most compact and luminous regions (like those of the central AGNs and star-forming clouds, which are typically warmer than the ambient material), particularly for strongly lensed sources with magnifications of $\mu>10$. Since differential lensing could bias measured CO SLEDs toward more high- $J$ CO emission, we also compute the median CO SLED as above, but restrict the included sources to those with magnifications of $\mu \leqslant 5$, where Serjeant (2012) found little evidence for differential lensing affecting the shape of galaxy-wide average CO SLEDs (Figure 4, top left). This selection reduces the number of lines in the sample by $\sim 1 / 3$. While removing the strongly lensed $\mu>5$ sources does systematically produce lower line ratios (at least where there are a significant number of input line measurements), differences in individual line ratios are not statistically significant (Table 3).

Galaxies' observed CO SLEDs can also be affected by the cosmic microwave background (CMB), which can both act as an additional heating mechanism and as a background above which the CO lines must be detected (e.g., da Cunha et al. 2013 and references therein). For galaxies at higher redshifts $(z \gtrsim 5)$, da Cunha et al. (2013) finds that the CMB's effect on theoretical CO SLEDs can be substantial. However, the effects on the measured line ratios also depend on the density and temperature of the molecular gas. We therefore cannot apply redshift-dependent corrections to individual galaxies in our sample since we do not have a priori constraints on their gas physical conditions. Therefore, our redshift-binned samples will have some additional scatter introduced by the effects of the CMB. However, the effects of the CMB in the redshift range of interest $(1 \leqslant z \leqslant 4)$ are generally much smaller than the scatter introduced by the galaxies' intrinsic range of gas temperatures and densities (da Cunha et al. 2013).

To ensure that including such a broad range of redshifts and IR luminosities will not bias our updated SLED, we also calculate the median line ratios for the sub-sample of galaxies from Carilli \& Walter (2013), Perna et al. (2018), etc., that match the redshift $(1 \leqslant z \leqslant 4)$, magnification $(\mu \leqslant 5)$, and IR luminosity $\left(0.1 \times 10^{12} L_{\odot} \leqslant L_{\mathrm{IR}} \leqslant 90 \times 10^{12} L_{\odot}\right)$ of our mid-IR sample described in Section 2. The median CO line ratios are reported in Table 3 and the CO SLED is plotted in Figure 4 (top right). There is no significant difference between the median line ratios for this sub-sample and the full literature sample.

Finally, we compare how the CO excitation of our IRselected sample compares with the CO SLED using the full literature sample. In Figure 4, we show the median CO SLED for all sources with $f(\mathrm{AGN})_{\mathrm{MIR}}$ (bottom left panel), and consider the sub-samples with IRS-determined $f(\mathrm{AGN})_{\text {MIR }}$ and color-determined $f(\mathrm{AGN})_{\text {MIR }}$ separately (bottom right panel; listed in Table 3). The sample with measured mid-IR AGN fractions has only $\sim 1-30 \mathrm{CO}$ detections per line, and no $\mathrm{CO}$ observations above the $\mathrm{CO}(7-6)$ transition. Again, we find no significant difference between the median $\mathrm{CO}$ line ratios for the $f(\mathrm{AGN})_{\mathrm{MIR}}$ sub-sample and the complete literature sample. We consider the effect of AGNs on the SLED in Section 4.2.

Though there is no statistically significant difference between the line ratios for the various median CO SLEDs, we use the median ratios calculated from the low-magnification sub-sample that match the redshift and IR luminosities of the sample of interest (top right of Figure 4) to avoid any potential 
Table 3

Median Brightness Temperature Ratios

\begin{tabular}{|c|c|c|c|c|c|c|c|c|c|c|c|c|}
\hline \multirow[b]{3}{*}{ Transition } & \multirow{2}{*}{\multicolumn{2}{|c|}{ All Sources }} & \multirow{2}{*}{\multicolumn{2}{|c|}{$\mu \leqslant 5$}} & \multirow{2}{*}{\multicolumn{2}{|c|}{ Matched $L_{\mathrm{IR}}, z, \mu$}} & \multirow{2}{*}{\multicolumn{2}{|c|}{ All }} & \multicolumn{4}{|c|}{ Measured $f(\mathrm{AGN})_{\mathrm{MIR}}$ Sample } \\
\hline & & & & & & & & & \multicolumn{2}{|c|}{ IRS-determined } & \multicolumn{2}{|c|}{ Color-determined } \\
\hline & Ratio & $N$ & Ratio & $N$ & Ratio & $N$ & Ratio $^{a}$ & $N$ & Ratio & $\bar{N}$ & Ratio & $\bar{N}$ \\
\hline $\mathrm{CO}(1-0)$ & $\ldots$ & 69 & $\ldots$ & 41 & $\ldots$ & 37 & $\ldots$ & 16 & $\ldots$ & 11 & $\ldots$ & 5 \\
\hline$r_{2,1}$ & $0.78 \pm 0.13$ & 73 & $0.59 \pm 0.17$ & 55 & $0.68 \pm 0.18$ & 38 & $0.86 \pm 0.30$ & 21 & $1.04 \pm 0.53$ & 15 & $0.97 \pm 0.55$ & 6 \\
\hline$r_{3,1}$ & $0.78 \pm 0.14$ & 108 & $0.62 \pm 0.18$ & 78 & $0.59 \pm 0.15$ & 75 & $0.65 \pm 0.21$ & 33 & $0.58 \pm 0.32$ & 19 & $0.67 \pm 0.31$ & 14 \\
\hline$r_{4,1}$ & $0.49 \pm 0.10$ & 54 & $0.36 \pm 0.11$ & 41 & $0.37 \pm 0.12$ & 29 & $0.38 \pm 0.12$ & 12 & $0.39 \pm 0.21$ & 5 & $0.38 \pm 0.13$ & 7 \\
\hline$r_{5,1}$ & $0.34 \pm 0.07$ & 41 & $0.28 \pm 0.11$ & 24 & $0.40 \pm 0.16$ & 11 & $0.36 \pm 0.19$ & 5 & $0.38 \pm 0.31$ & 2 & $0.31 \pm 0.19$ & 3 \\
\hline$r_{6,1}$ & $0.31 \pm 0.06$ & 47 & $0.26 \pm 0.08$ & 32 & $0.22 \pm 0.07$ & 11 & $0.28 \pm 0.09$ & 2 & $\ldots$ & 0 & $0.24 \pm 0.08$ & 2 \\
\hline$r_{7,1}$ & $0.21 \pm 0.04$ & 37 & $0.19 \pm 0.08$ & 21 & $0.15 \pm 0.07$ & 11 & $0.21 \pm 0.10$ & 5 & $0.20 \pm 0.15$ & 3 & $0.24 \pm 0.09$ & 2 \\
\hline$r_{8,1}$ & $0.11 \pm 0.03$ & 13 & $0.08 \pm 0.04$ & 5 & $0.08 \pm 0.04$ & 5 & $\ldots$ & 0 & $\ldots$ & 0 & $\cdots$ & 0 \\
\hline$r_{9,1}$ & $0.14 \pm 0.04$ & 12 & $0.13 \pm 0.04$ & 5 & $0.12 \pm 0.04$ & 4 & $\ldots$ & 0 & $\ldots$ & 0 & $\ldots$ & 0 \\
\hline$r_{10,1}$ & $0.08 \pm 0.04$ & 7 & $0.12 \pm 0.06$ & 2 & $0.07 \pm 0.02$ & 1 & $\ldots$ & 0 & $\ldots$ & 0 & $\ldots$ & 0 \\
\hline$r_{11,1}$ & $0.12 \pm 0.03$ & 7 & $0.09 \pm 0.03$ & 3 & $0.08 \pm 0.03$ & 3 & $\ldots$ & 0 & $\ldots$ & 0 & $\ldots$ & 0 \\
\hline
\end{tabular}

Notes. The first three sets of median line ratios are calculated from the parent sample of Carilli \& Walter (2013), Pope et al. (2013), Aravena et al. (2014), Sharon et al. (2016), Yang et al. (2017), Frayer et al. (2018), Perna et al. (2018), and references therein (which includes our sample with calculated mid-IR AGN fractions), and various subsets as indicated by the columns' titles. Median line ratios for our sample with measured mid-IR AGN fractions are shown in the last three columns. In all cases, $N$ refers to the number of galaxies with detections of the specified $\mathrm{CO}$ transition that contribute to the median line luminosity, and thus the median line ratio. ${ }^{\mathrm{a}}$ The astute reader may notice that combined $f(\mathrm{AGN})_{\mathrm{MIR}}$ median ratios do not always fall between the color-determined and IRS-determined median ratios (similar effects are also seen for other ratios presented in this paper). This result is due to our method of calculating a ratio of medians as opposed to a median of ratios as described in Section 3.4.1 (which is necessary since not all sources have detections of both the relevant lines and we want to use all available information). Since we calculate the median $L_{\mathrm{CO}(J-(J-1))}^{\prime}$ and the median $L_{\mathrm{CO}(1-0)}^{\prime}$, those luminosities may skew in different directions depending on the number of detections and luminosities of their sub-populations. Thus the resulting ratio of median luminosities may be higher or lower than that of the objects or sub-samples from which the ratio is composed.

biases when extrapolating down from higher- $J \mathrm{CO}$ lines to the $\mathrm{CO}(1-0)$ line luminosity. The highest- $J$ transition we use to determine the ground state $\mathrm{CO}$ line luminosity is the $\mathrm{CO}(4-3)$ line. We use these excitation-corrected CO line luminosities to determine molecular gas masses.

\subsubsection{Choice of CO-to- $\mathrm{H}_{2}$ Conversion Factor}

Calculating gas mass from $L_{\mathrm{CO}}^{\prime}$ requires assuming a conversion factor, $\alpha_{\mathrm{CO}}$, that depends on the conditions in the ISM. These assumptions range from using a single conversion factor for all galaxies (e.g., Scoville et al. 2016), to a bimodal conversion for the main sequence and starbursts (e.g., Bolatto et al. 2013), to a continuous conversion factor that depends on CO surface density and metallicity (e.g., Narayanan et al. 2012). We explore two extremes: calculating gas mass with a single conversion factor and using a physically motivated continuous conversion factor from Narayanan et al. (2012).

The continuous conversion factor requires us to know the metallicity and the surface area of the ISM in our galaxies. For this literature-based sample, most $\mathrm{CO}$ observations are unresolved. However, the shape of the far-IR SED contains information about the physical extent of the dust emission; at the most fundamental level, the luminosity of a blackbody depends solely on temperature and radius. We make the simplifying assumption that the dust and gas are predominantly cospatial (though the few existing high-resolution dust studies may challenge that assumption; e.g., Hodge et al. 2015, 2016, 2019; Tadaki et al. 2017). We use the radius output by the Chakrabarti \& McKee (2005) code to calculate surface area.

The continuous $\alpha_{\mathrm{CO}}$ conversion factor is given by

$$
\alpha_{\mathrm{CO}}=\frac{0.7 * \Sigma_{\mathrm{CO}}^{-0.32}}{Z^{0.65}}
$$

where $\Sigma_{\mathrm{CO}}$ is the $\mathrm{CO}$ surface density in $\mathrm{K} \mathrm{km} \mathrm{s}^{-1}$ and $Z$ is the metallicity (Narayanan et al. 2012). To calculate the CO surface density, we use the characteristic radius from the far-IR fitting code (listed in Table 2) and calculate an average surface density given by $\Sigma_{\mathrm{CO}}=L_{\mathrm{CO}}^{\prime} / \pi R^{2}$. Since we lack stellar masses for many galaxies, we cannot explicitly use the massmetallicity relationship to estimate metallicities. However, $M_{\text {dust }}$ also correlates with metallicity, so we follow the calibration in Rémy-Ruyer et al. (2015):

$$
\begin{aligned}
\log (\mathrm{O} / \mathrm{H})+12.0 & =\frac{\log M_{\text {dust }}+15.0}{2.6} \\
Z & =10^{(\log (\mathrm{O} / \mathrm{H})+12.0)-8.69}
\end{aligned}
$$

(where 8.69 is the value for solar metallicity). For the above expression, we use $M_{\text {dust }}^{\mathrm{CM}}$ (rather than $M_{\text {dust }}^{\mathrm{S}}$ ) to be consistent with our calculated radius. This method yields CO-to- $\mathrm{H}_{2}$ conversion factors of $0.08<\alpha_{\mathrm{CO}}<0.73$ (in units of $M_{\odot}\left(\mathrm{K} \mathrm{km} \mathrm{s}^{-1} \mathrm{pc}^{2}\right)^{-1}$; Table 2) and a mean value of $0.25 M_{\odot}\left(\mathrm{K} \mathrm{km} \mathrm{s}^{-1} \mathrm{pc}^{2}\right)^{-1}$. All of the conversion factors calculated in this manner are less than the $\alpha_{\mathrm{CO}}=0.8$ determined for local dusty starbursts (Solomon et al. 1997) and frequently applied to high- $z$ starbursts. The relatively low conversion factors are likely a product of the compact dust sizes we use to calculate the $\mathrm{CO}$ surface brightness. However, without spatially resolved $\mathrm{CO}$ observations, the size of the dust-emitting region is the best estimate for the $\mathrm{CO}$ size currently available for this sample. See Section 4.3 for further discussion.

As a simple upper limit on the gas masses, we also assume a constant conversion factor of $\alpha_{\mathrm{CO}}=4.6$ (Solomon \& Barrett 1991), which is standard for main-sequence galaxies. We do not know if our galaxies are on the main sequence as 

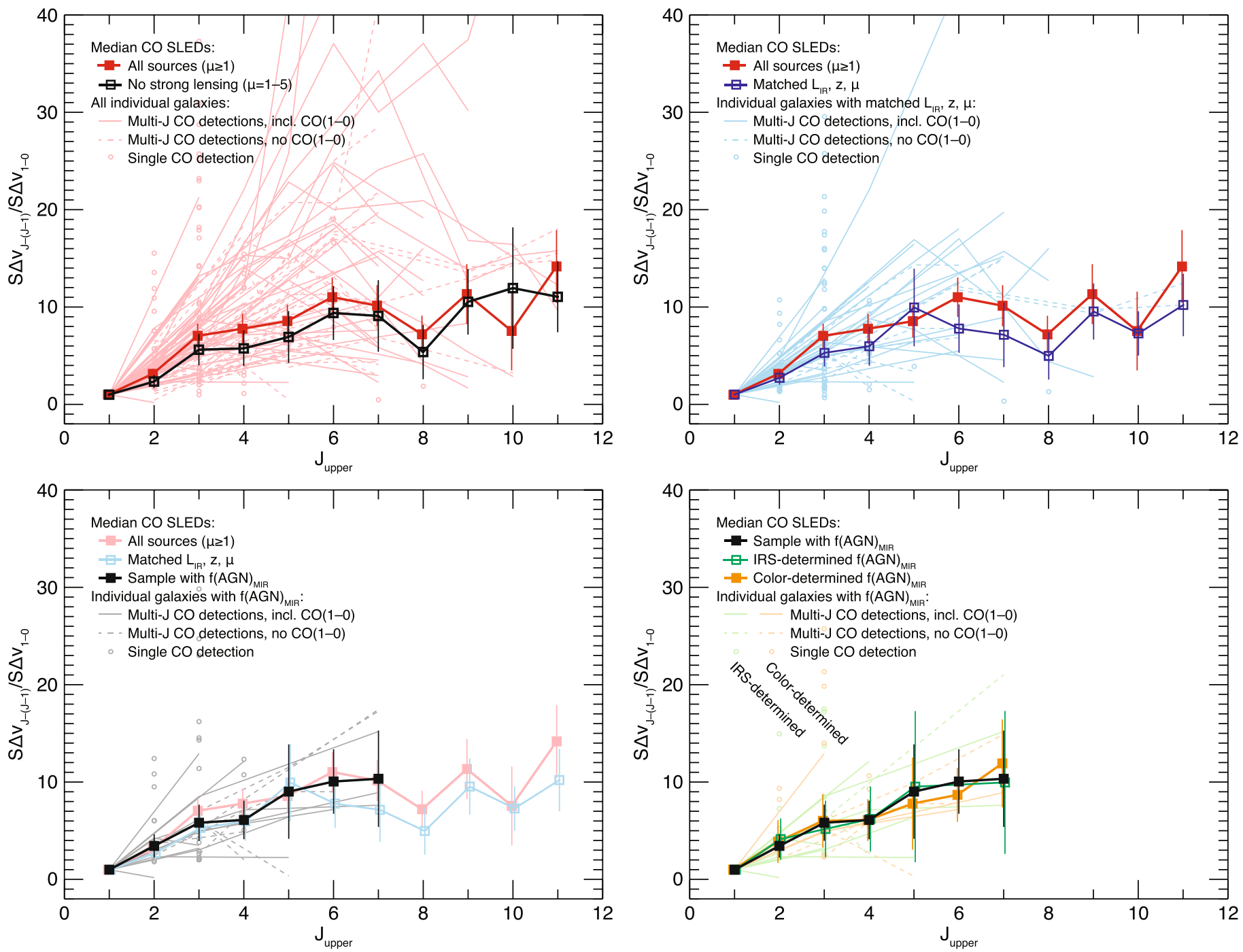

Figure 4. Median CO SLEDs (squares and thick lines) and the SLEDs of the individual contributing galaxies (thin lines). Sources with only a single reported CO detection are shown as circles; the SLEDs of sources that lack published $\mathrm{CO}(1-0)$ measurements are shown as thin dashed lines. All sources that lack a $\mathrm{CO}(1-0)$ detections are scaled by the median $\mathrm{CO}(1-0)$ flux of the population as described in Section 3.4, and therefore their normalizations may change slightly from panel to panel (this does not affect the median CO SLEDs). Top left: the median CO SLED and line ratio uncertainties for the full sample from Carilli \& Walter (2013) (red/ solid squares; individual contributing sources in pink) and for the sub-sample with $1 \leqslant \mu \leqslant 5$ (black/open squares). Top right: we repeat the full sample's SLED (red/solid squares) for reference and show the median SLED for the sub-sample within the matching range of far-IR luminosities, redshifts, and magnification factors as the IR galaxies of interest in this work (blue/open squares; individual contributing galaxies in light blue). Bottom left: we repeat the full sample's SLED (light red/ solid squares) and matched sample's SLED (light blue/open squares), and show the median SLED for the galaxies with measured $f(\mathrm{AGN})_{\mathrm{MIR}}$ (black/solid squares; individual contributing galaxies in gray). Bottom right: we repeat the median SLED for all galaxies with $f(\mathrm{AGN})_{\mathrm{MIR}}$ (black/solid squares), and show the median SLEDs for the galaxies with IR AGN fractions determined from IRS spectra (green/open squares; individual contributing galaxies in light green) and the those determined from color templates (orange/solid squares; individual contributing galaxies in light orange).

defined by $\mathrm{SFR} / M_{*}$, since we do not have uniform $M_{*}$ measurements. Just based on $L_{\mathrm{IR}}$, Sargent et al. (2012) find that at $z \sim 1,90 \%$ of galaxies with $L_{\mathrm{IR}}<3 \times 10^{11} L_{\odot}$ are on the main sequence, as are $50 \%$ of galaxies with $L_{\mathrm{IR}}<2 \times$ $10^{12} L_{\odot}$. Similarly, at $z=2,90 \%$ of galaxies with $L_{\mathrm{IR}}<$ $1.5 \times 10^{12} L_{\odot}$ are on the main sequence, as are $50 \%$ of galaxies with $L_{\mathrm{IR}}<8 \times 10^{12} L_{\odot}$. Given these luminosity estimates, our sample is likely a mix of main sequence and starbursting galaxies. Since we cannot distinguish between these for this sample, we use the main-sequence conversion factor, although any observed trends would be unchanged if we instead used $\alpha_{\mathrm{CO}}=0.8$. We calculate $M_{\text {gas }}^{\mathrm{V}}$ with the varying $\alpha_{\mathrm{CO}}$ and $M_{\mathrm{gas}}^{\mathrm{MS}}$ with $\alpha_{\mathrm{CO}}=4.6$ and list both in Table 2.

\section{Analysis and Discussion}

\subsection{SFE in AGNs}

In the past, galaxies have conventionally been categorized in terms of two different modes of star formation: a main-sequencelike "normal" mode (historically associated with local disk galaxies) and a starburst mode (historically associated with local ultra/luminous infrared galaxies (U/LIRGs) and frequently extended to high- $z$ SMGs). Normal SFGs have long gas depletion timescales of $>100 \mathrm{Myr}$ (or for the depletion timescale's inverse: high SFEs), while starbursts are compact and have short gas depletion timescales. However, due to increased gas fractions, the relationship between compactness, 


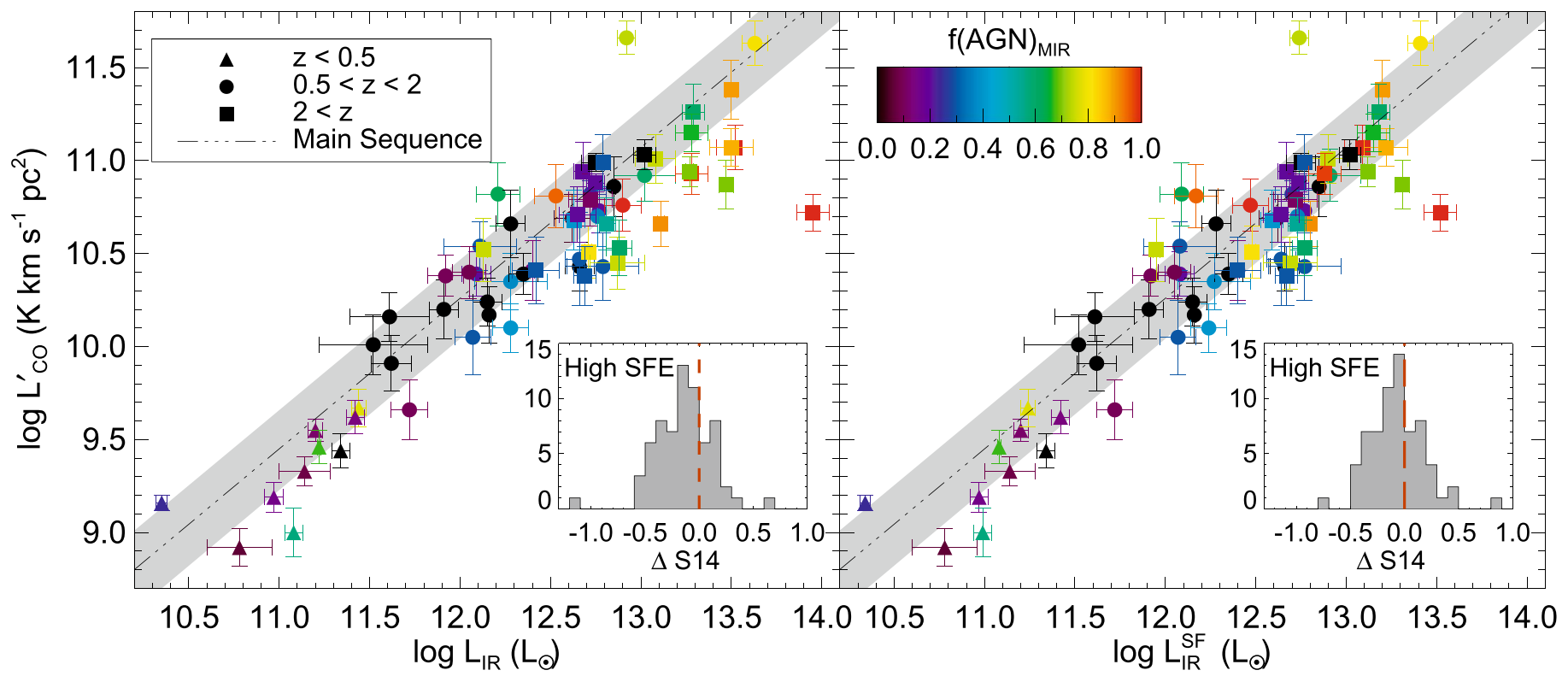

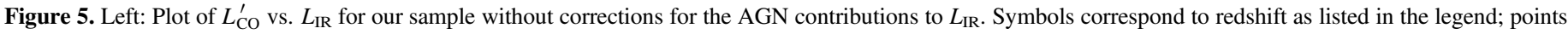

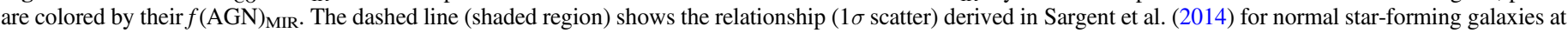

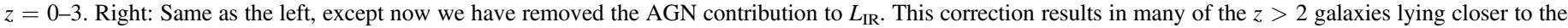

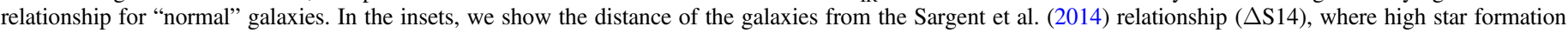

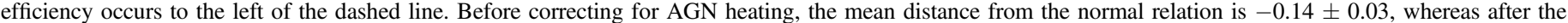
correction, the mean is $-0.07 \pm 0.03$ - a small but statistically significant shift.

main sequence, starburst, and SFE becomes increasingly murky with lookback time (e.g., Kartaltepe et al. 2012; Chang et al. 2018). For example, in a sample of $z<1$ IR-selected galaxies, Kirkpatrick et al. (2014) demonstrate that the SFR/ $M_{*}$ criterion does not correlate with SFE. In addition, typical assumptions of bimodal $\mathrm{CO}-$ to- $\mathrm{H}_{2}$ conversion factors may induce a separation between normal galaxies and starbursts (e.g., Daddi et al. 2010), and hinges on distinguishing reliably between a starburst galaxy and a normal galaxy, rather than assuming a smooth transition between the two regimes. In order to avoid the complicating choice of conversion factor, Carilli \& Walter (2013) use a standard convention of comparing $L_{\mathrm{IR}}$ with $L_{\mathrm{CO}}^{\prime}$ as a proxy for SFE, where starbursting galaxies have higher SFEs manifested as an elevated $L_{\mathrm{CO}}^{\prime}\left(\propto M_{\text {gas }}\right)$ for a given $L_{\mathrm{IR}}(\propto \mathrm{SFR})$ (see also Daddi et al. 2010; Genzel et al. 2010; Sargent et al. 2014). In Carilli \& Walter (2013), radio AGNs and QSOs had higher $L_{\mathrm{IR}} / L_{\mathrm{CO}}^{\prime}$ than main-sequence-selected galaxies.

In light of our recalculated homogeneous $L_{\mathrm{IR}}$ and $L_{\mathrm{CO}}^{\prime}$ values, and our quantitative/continuous approach to AGN emission, we revisit the relationship between $L_{\mathrm{IR}}$ and $L_{\mathrm{CO}}^{\prime}$ in Figure 5. We compare our sample to the $L_{\mathrm{IR}}-L_{\mathrm{CO}}^{\prime}$ correlation for normal star formation in $z=0-3$ galaxies from Sargent et al. (2014). We should take care here to note that this relationship is not a main sequence of star formation in the way that the relationship between SFR and $M_{*}$ is (e.g., Noeske et al. 2007). Rather, it is the region of parameter space where most main-sequence galaxies (the so-called normal population) tend to lie. But a galaxy in the gray regime in Figure 5 may not necessarily be a main-sequence galaxy according to $\mathrm{SFR} / M_{*}$. Many of our $z>2$ galaxies lie off this normal relationship in the area of the graph (lower right) that would indicate a high SFE. This is not surprising since high- $z$ observations tend to be biased toward the brightest systems. As the relationship between $L_{\mathrm{CO}}^{\prime}$ and $L_{\mathrm{IR}}$ is meant to be a proxy for the relationship between molecular gas mass and SFR (i.e., an integrated form of the Schmidt-Kennicutt relation; Schmidt 1959; Kennicutt 1989), we should only be using the portion of $L_{\mathrm{IR}}$ due to star formation, $L_{\mathrm{IR}}^{\mathrm{SF}}$. We therefore show $L_{\mathrm{IR}}^{\mathrm{SF}}$ versus $L_{\mathrm{CO}}^{\prime}$ in the right panel of Figure 5 . Using $L_{\mathrm{IR}}^{\mathrm{SF}}$, almost every galaxy lies around the normal relation, which we quantify by calculating the difference in observed $L_{\mathrm{CO}}^{\prime}$ from the Sargent et al. (2014) prediction for normal star formation given a galaxy's $L_{\mathrm{IR}}^{\mathrm{SF}}$. Before the AGN correction, the geometric mean difference $\left(\log L_{\mathrm{CO}}^{\prime}-\log L_{\mathrm{CO}}^{\prime}(\mathrm{S} 14)\right)$ is $-0.14 \pm 0.03$, where the negative indicates that sources are underluminous in CO, corresponding to a higher SFE. After the AGN correction, the mean difference is $-0.07 \pm 0.03$; although it is small, this shift indicates how the presence of an AGN can bias interpretations of galaxy relationships if not properly accounted for.

This result is noticeably differs from the recent work of Perna et al. (2018) which finds that obscured and unobscured QSOs from Carilli \& Walter (2013) have higher SFEs. Of their sample, 30 SMGs, 35 obscured quasars, and six unobscured quasars meet our selection criteria of being unlensed, at $z<4$, and having a $\mathrm{CO}$ detection. Of these, only 15 SMGs, 18 obscured QSOs, and one unobscured QSO meet our criteria of having enough publicly available IR photometry to fully sample the SED. (All except three of these galaxies are drawn from the Carilli \& Walter (2013) compilation.) Perna et al. (2018) determine $L_{\mathrm{IR}}$ using a spectral decomposition method that relies on the Chary \& Elbaz (2001) and Dale \& Helou (2002) templates derived from local galaxies. As we demonstrated in Kirkpatrick et al. (2012), IR-luminous galaxies at $z>1$ have significantly different far-IR emission than the Chary \& Elbaz (2001) library and require their own set of templates. In Figure 6, we show that the measurements from Perna et al. (2018) do indeed place QSOs in the region of high SFE. However, when calculating $L_{\mathrm{IR}}$ using high- $z$ templates that account for AGN emission and calculating $L_{\mathrm{CO}}^{\prime}$ using our new CO SLEDs, the same QSOs lie much closer to the main 


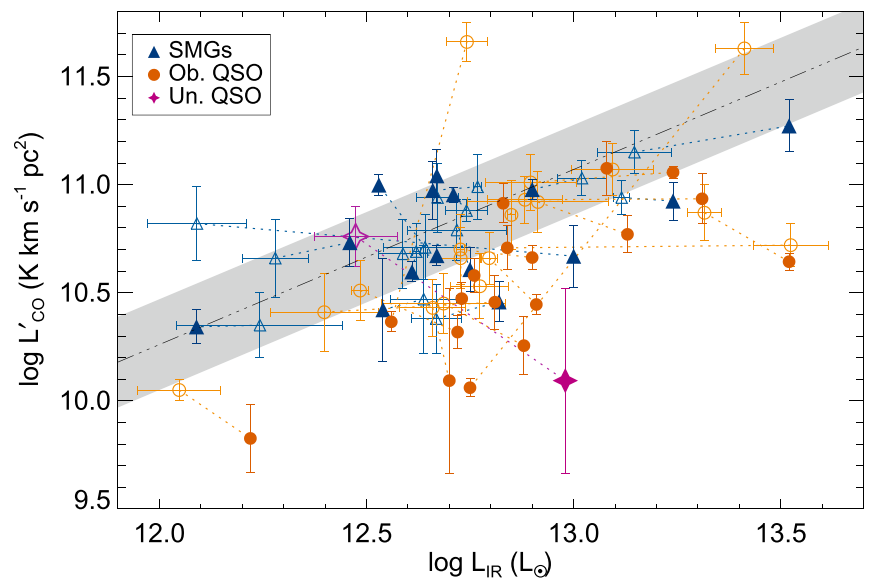

Figure 6. Comparison of the Perna et al. (2018) measurements for submillimeter galaxies (filled blue triangles), obscured quasars (filled orange circles) and unobscured quasars (filled purple star) with our measurements for those same sources (unfilled symbols, connected with dotted lines) on the $L_{\mathrm{IR}}-L_{\mathrm{CO}}^{\prime}$ relation. We have remeasured $L_{\mathrm{IR}}$ using high- $z$ templates and accounting for AGN emission, and we have created new CO SLEDs to calculate $L_{\mathrm{CO}}^{\prime}$ from excited transitions. The different measurements can cause different interpretations of the role AGNs play in galaxy evolution. The dashed line and shaded region is from Sargent et al. (2014) as in Figure 5.

sequence. This result clearly illustrates that conclusions regarding how galaxies evolve critically depend on how the measurements are made.

In Figure 7, we look for a correlation between black hole heating of the ISM and depressed SFE. We parameterize SFE as $L_{\mathrm{IR}}^{\mathrm{SF}} / L_{\mathrm{CO}}^{\prime}$. We first use the Sargent et al. (2014) relation in Figure 5 to determine the SFE of normal galaxies. Then, we calculate the distance of our galaxies from this relation, $\Delta \mathrm{SFE}_{\mathrm{S} 14}$. We plot $\Delta \mathrm{SFE}_{\mathrm{S} 14}$ as a function of $f(\mathrm{AGN})_{\mathrm{MIR}}$ in Figure 7 . The shaded region represents a factor of 3 above and below the Sargent et al. (2014) SFE. There is no trend between $\triangle \mathrm{SFE}_{\mathrm{S} 14}$ and $f(\mathrm{AGN})_{\mathrm{MIR}}$ as confirmed by a Spearman's rank test $(\rho=-0.02$, significance $=0.85$, which is not a statistically significant deviation from 0.0$)$.

We split the sample at $f(\mathrm{AGN})_{\mathrm{MIR}}=0.5$ to measure whether low $f(\mathrm{AGN})_{\mathrm{MIR}}$ galaxies have a different distribution of $\triangle \mathrm{SFE}_{\mathrm{S} 14}$ than high $f(\mathrm{AGN})_{\text {MIR }}$ galaxies. We plot the distributions in the right panel of Figure 7. A two-population Kolmogorov-Smirnov test cannot rule out the null hypothesis that $\triangle \mathrm{SFE}_{\mathrm{S} 14}$ of the two subsamples are drawn from the same parent population. In other words, for our limited, heterogenous sample, there is no significant difference in the SFEs of high $f(\mathrm{AGN})_{\text {MIR }}$ galaxies versus low $f(\mathrm{AGN})_{\text {MIR }}$ galaxies. However, if we had not corrected $L_{\mathrm{IR}}$ for the AGN contribution when calculating SFE and $\triangle \mathrm{SFE}_{\mathrm{S} 14}$, the two-population Kolmogorov-Smirnov test indicates that the distributions of $\triangle \mathrm{SFE}_{\mathrm{S} 14}$ for the SF-dominated and AGNdominated galaxies significantly differ. We show the uncorrected $\triangle \mathrm{SFE}_{\mathrm{S} 14 \mathrm{~S}}$ as the unfilled symbols in the left panel, and as the purple histogram in the right panel of Figure 7.

The SFE assumes that star formation is the dominant mode of consuming gas. Powerful AGNs are also accreting gas, but at a much lower rate (e.g., Delvecchio et al. 2014). Massive outflows could expel gas, but estimates of this mass-loss rate exist in only a handful of galaxies. In the local universe, an ionized mass-loss rate of $2 M_{\odot} \mathrm{yr}^{-1}$ was measured in a Compton-thick quasar (Revalski et al. 2018) and that outflow was solely attributed to its AGN (as opposed to a potential nuclear starburst); such outflow rates are not enough to drastically alter gas depletion timescales in AGNs relative to normal SFGs. Gowardhan et al. (2018) find massive molecular outflows of hundreds of solar masses per year in two lowredshift $(z<0.15)$ ULIRGs. In these galaxies, the molecular outflow is the dominant source depleting the gaseous ISM. However, the outflow correlates with both SFR and AGN luminosity, indicating that the AGN alone is not the dominant predictor of quenching-enabling feedback.

The fact that we do not observe lower SFE in AGNs has three possible interpretations. First, our sample may be biased. Considering we have put together a heterogeneous, data-mined sample, we may be missing a key portion of galaxies that would exhibit enhanced or depleted SFE with increasing AGN energetics. However, for the sample that we have, we have taken care to measure properties and identify AGNs in a homogeneous way. A large, blind IR survey followed by CO observations for all galaxies is the proper way to test gas depletion timescales in IR AGNs.

A second potential reason for the lack of difference between the SFEs may be that IR AGNs do not occur at special points in galaxies' life cycles. (Kirkpatrick et al. 2012) showed that AGNs are heating the central ISM relative to normal SFGs (indicated by rising warm dust emission from $\lambda=10-100 \mu \mathrm{m}$; see also some discussion of AGN heating effects on $\mathrm{CO}$ in the following subsection). This heating may prevent star formation in the central $100 \mathrm{pc}$, and could be a significant contributor to quenching; verification of this effect requires future resolved observations of the ISM in the centers of IR AGN hosts. However, the lack of evidence for lower gas masses or shorter gas depletion timescales in our AGNs suggests that AGNs do not play a universal role in quenching star formation, even in massive galaxies (see also $\mathrm{Xu}$ et al. 2015). It leaves open the question of whether AGNs are growing in lockstep with their galaxies, consuming the same fuel as the nascent stars (e.g., Kormendy \& Ho 2013 and references therein). If AGNs are passively accreting in their hosts, rather than actively quenching, then why do only some galaxies have visible AGNs? Either the triggering mechanisms of IR AGNss do not seem to be universally responsible for enhanced star formation in dusty SFGs, as evidenced by our galaxies with high SFRs, short $\tau_{\text {dep }}$, but no AGN signatures, or the physics associated with AGN flickering make measuring any correlation hopeless.

Finally, we consider the possibility that the lack of difference between the SFEs may be rooted in the timescales of AGN activity. Theoretical models suggest that supermassive black holes go through growth spurts, causing a variable luminosity on 10,000 yr timescales (Hickox et al. 2014) that would make correlations between AGN activity and SFR (or SFE) difficult to identify. In addition, the density of dust clouds in the torus cause it to cool quickly, from 1000 to $100 \mathrm{~K}$, in less than $10 \mathrm{yr}$, (Ichikawa \& Tazaki 2017), making IR AGNs unidentifiable when the supermassive black hole is not actively accreting. For such variable AGNs, we may not expect to see a correlation between $f(\mathrm{AGN})_{\mathrm{MIR}}$ and SFE. The exact timescales traced by AGNs' IR emission is relatively unconstrained, but it is likely extremely short ( $\ll 1 \mathrm{Myr}$ ). IR-determined SFRs trace timescales of $100 \mathrm{Myr}$, so we may never see a correlation between the two phenomena, even if there is an underlying relationship. Finally, galaxies evolve over Gyr timescales. Given these differing ranges, looking for correlations between SFE and AGN activity may never reveal any information about the impact of AGN activity on its host galaxy. 


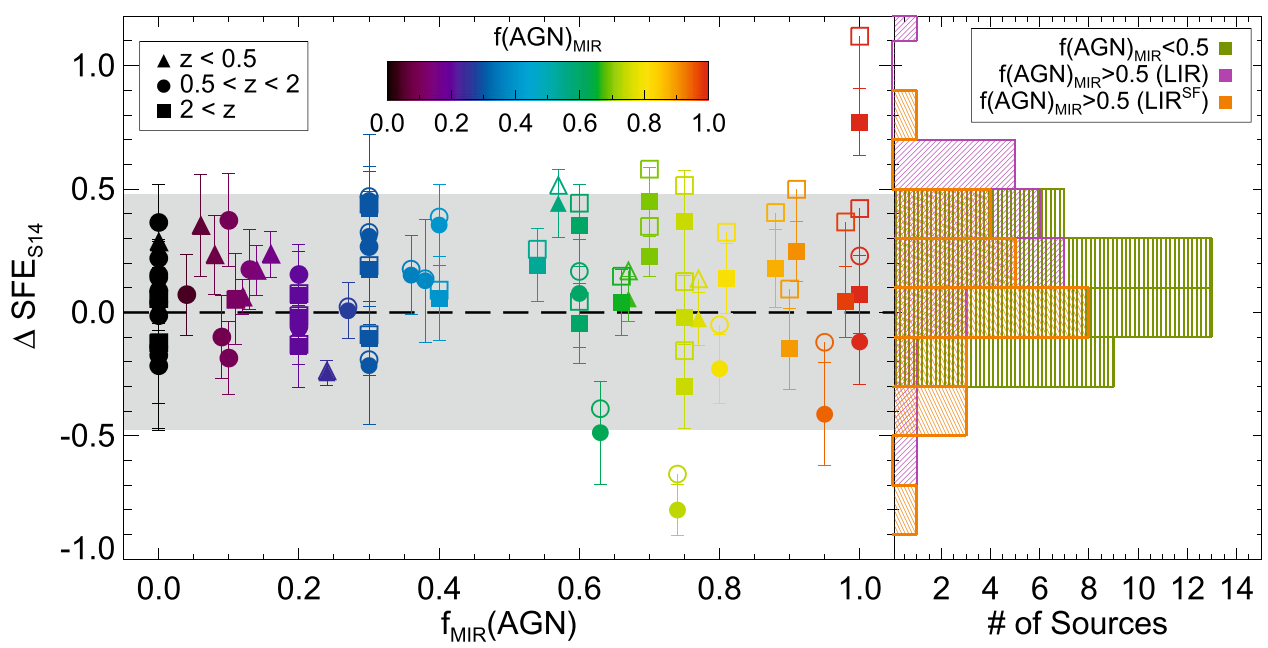

Figure 7. We look for a possible correlation between SFE (as parameterized by $L_{\mathrm{IR}}^{\mathrm{SF}} / L_{\mathrm{CO}}^{\prime}$ ) and AGN strength. We calculate the difference in SFE from the relationship measure by Sargent et al. (2014), shown in Figure 5, and plot $\Delta \mathrm{SFE}_{\mathrm{S} 14}$ vs. $f(\mathrm{AGN})_{\mathrm{MIR}}$ in the left panel. The shaded region represents a factor of 3 difference from the Sargent et al. (2014) relationship. The right panel shows the distribution of galaxies with $f(\mathrm{AGN})_{\mathrm{MIR}}<0.5$ (green) and $f(\mathrm{AGN})_{\mathrm{MIR}} \geqslant 0.5$ (orange). There is no trend of increasing or decreasing SFE with increasing $f(\mathrm{AGN})_{\mathrm{MIR}}$. If we had not corrected $L_{\mathrm{IR}}$ for AGN emission, there would be a trend, as exhibited by the unfilled symbols in the left panel and the purple histogram in the right panel.

Given the limitations potentially introduced by the timescales of AGN activity, we briefly consider whether progress might be made using future far-IR observations. As a back-ofthe-envelope example, we assume the AGN heating can account for all dust emission at $\lambda \sim 70 \mu \mathrm{m}$ in a galaxy with $\nu$ $L_{\nu}=10^{12} L_{\odot}$ at $\lambda=70 \mu \mathrm{m}$. If we assume that an AGN behaves as a compact emission source surrounded by a sphere of dust, then we can estimate the radius using $L=4 \pi \sigma R^{2} T^{4}$. We use $T=100 \mathrm{~K}$, which gives $R \sim 100 \mathrm{pc}$. This is similar to the radius of the NLR, which extends 10-1000 pc (Groves et al. 2006; Hainline et al. 2013). At the extreme, if the AGN is heating the local diffuse cold neutral medium, beyond the ionized NLR, the main cooling is through the [C II] finestructure line. For thermal equilibrium, with a low density of $n=20 \mathrm{~cm}^{-3}$, the cooling timescale is longer than $10,000 \mathrm{yr}$. However, if the AGN's influence is confined to the ionized NLR, then the cooling timescale will be much shorter (Groves et al. 2006; Hainline et al. 2016). Therefore, resolved $70 \mu \mathrm{m}$ observations (on scales of a kpc or less) may be a promising way to look for AGN emission over long timescales.

\subsection{Average Gas Excitation Properties of AGN- and Starbust- dominated Galaxies}

Using the methods described in Section 3.4, we also calculate the median CO SLEDs for galaxies with measured mid-IR AGN fractions (Table 4 and Figure 8 ). While the effects of AGN heating are likely continuous with $f(\mathrm{AGN})_{\mathrm{MIR}}$, there are too few $\mathrm{CO}$ detection per source to look for fine-grained differences in the CO SLEDs as a function of $f(\mathrm{AGN})_{\mathrm{MIR}}$. Therefore, as in the previous section, we divide the sources into two groups at $f(\mathrm{AGN})_{\mathrm{MIR}}=0.5$ in order to improve our statistics; for convenience, we refer to the sources below and above $f(\mathrm{AGN})_{\mathrm{MIR}}=0.5$ as SF-dominated and AGN-dominated respectively. In the bottom panel of Figure 8, we compare our new SLEDs to previous work (SLEDs for local ULIRGs are taken from Kamenetzky et al. 2016). Our median SF-dominated SLED closely resembles the average SMG SLED from Bothwell et al. (2013) and is also consistent with
Table 4

Median Line Ratios for AGN- and SF-dDominated Galaxies

\begin{tabular}{|c|c|c|c|c|}
\hline \multirow[b]{2}{*}{ Transition } & \multicolumn{2}{|c|}{$f(\mathrm{AGN})_{\mathrm{MIR}}<50 \%$} & \multicolumn{2}{|c|}{$f(\mathrm{AGN})_{\mathrm{MIR}} \geqslant 50 \%$} \\
\hline & Ratio & $N$ & Ratio & $N$ \\
\hline $\mathrm{CO}(1-0)$ & $\ldots$ & 8 & $\ldots$ & 8 \\
\hline$r_{2,1}$ & $0.61 \pm 0.15$ & 16 & $1.27 \pm 1.01$ & 5 \\
\hline$r_{3,1}$ & $0.52 \pm 0.17$ & 15 & $0.92 \pm 0.44$ & 18 \\
\hline$r_{4,1}$ & $0.29 \pm 0.09$ & 7 & $0.76 \pm 0.34$ & 5 \\
\hline$r_{5,1}$ & $0.21 \pm 0.10$ & 2 & $0.74 \pm 0.43$ & 3 \\
\hline$r_{6,1}$ & $0.21 \pm 0.05$ & 1 & $0.52 \pm 0.23$ & 1 \\
\hline$r_{7,1}$ & $0.14 \pm 0.03$ & 1 & $0.54 \pm 0.24$ & 4 \\
\hline
\end{tabular}

the SLEDs of local U/LIRGs such as Arp 220 and M82 (given our large uncertainties and the effects of small-number statistics). Our median AGN-dominated SLED appears consistent with thermalized emission in the Rayleigh-Jeans limit (where flux ratios are equal to $J^{2}$ ). While thermalized emission is expected to arise in the dense molecular ISM of ULIRGs, SMGs, and AGNs for low- $J$ lines (e.g., Weiß et al. 2007; Harris et al. 2010; Riechers et al. 2011a), it is generally not expected for the kinetic temperature to be sufficiently high to drive higher- $J$ emission to the asymptotic Rayleigh-Jeans limit. The consistency with thermalized emission for the high- $J$ lines in the AGN-dominated sample is likely due to the small-number statistics that produce the considerable uncertainties. Significant amounts of high excitation emission are not uncommon for high-z AGN, and our AGN-dominated SLED is consistent with that of the Cloverleaf galaxy and IRAS F10214+4724, which are both type 1 QSOs at $z>2$. Local U/LIRGs with known AGNs (i.e., NGC 6240, Mrk 231, and NGC 253) appear to have somewhat lower line ratios in their highest- $J$ transitions than we find in our median AGN-dominated SLED, but again they are consistent given our sample's small numbers and large uncertainties. Given the uncertainties for our line ratios (Table 4), there is no significant difference between the $\mathrm{CO}$ excitation for the AGN-dominated galaxies $\left(f(\mathrm{AGN})_{\mathrm{MIR}} \geqslant 0.5\right)$ and the SF-dominated galaxies $\left(f(\mathrm{AGN})_{\mathrm{MIR}}<0.5\right)$. 

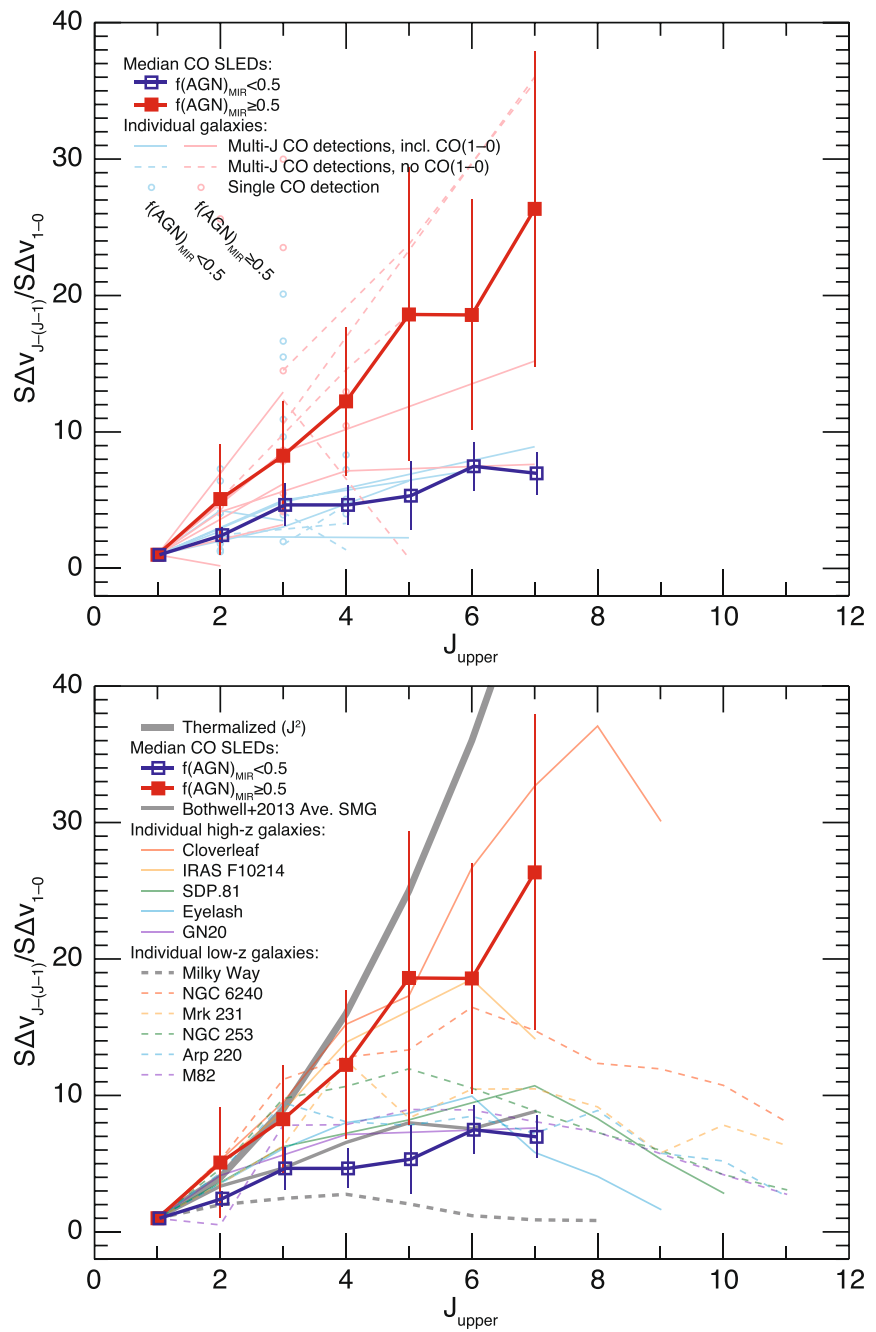

Figure 8. Median CO SLEDs and line ratio uncertainties for sources with midIR AGN fractions $\geqslant 50 \%$ (red filled squares/thick lines) and mid-IR AGN fractions $<50 \%$ (dark blue open squares/thick lines). Top: the CO detections for individual galaxies contributing each median CO SLED are shown in light red circles/thin lines for the $\geqslant 50 \%$ mid-IR AGN fractions and light blue circles/thin lines for the $<50 \%$ mid-IR AGN fractions. Sources with only a single reported $\mathrm{CO}$ detection are shown as light color circles; the SLEDs of sources that lack published $\mathrm{CO}(1-0)$ measurements are shown with dashed lines (both scaled by the corresponding sub-samples' median $\mathrm{CO}(1-0)$ fluxes as described in Section 3.4). Bottom: we compare the same median CO SLEDs in the top panel to those of several well-known high-redshift galaxies (solid colored thin lines) and low-redshift galaxies (dashed colored thin lines) as listed in the legend. CO SLEDs for the high-redshift galaxies are taken from the samples described in Section 3.4.1 that are used to calculate gas mass excitation corrections. CO SLEDs for low-redshift sources are taken from Kamenetzky et al. (2016), except for the Milky Way (dashed gray line) which is from Fixsen et al. (1999). We also show the average SLED for SMGs calculated in Bothwell et al. (2013) (solid gray line), and the line ratios for thermalized emission (thick gray line).

However, there may be a systematic difference in the $\mathrm{CO}$ SLED for the two populations (Figure 8) even if individual line ratios are consistent within their uncertainty. In order to explore whether this systematic difference is significant, we compare the average emission line flux between the two populations for each CO line directly, thereby avoiding additional uncertainty from the $\mathrm{CO}(1-0)$ line when comparing the ratios between the two populations. We use a similar bootstrapping with replacement technique as described in Section 3.4 to determine the median line luminosities and their uncertainties for both

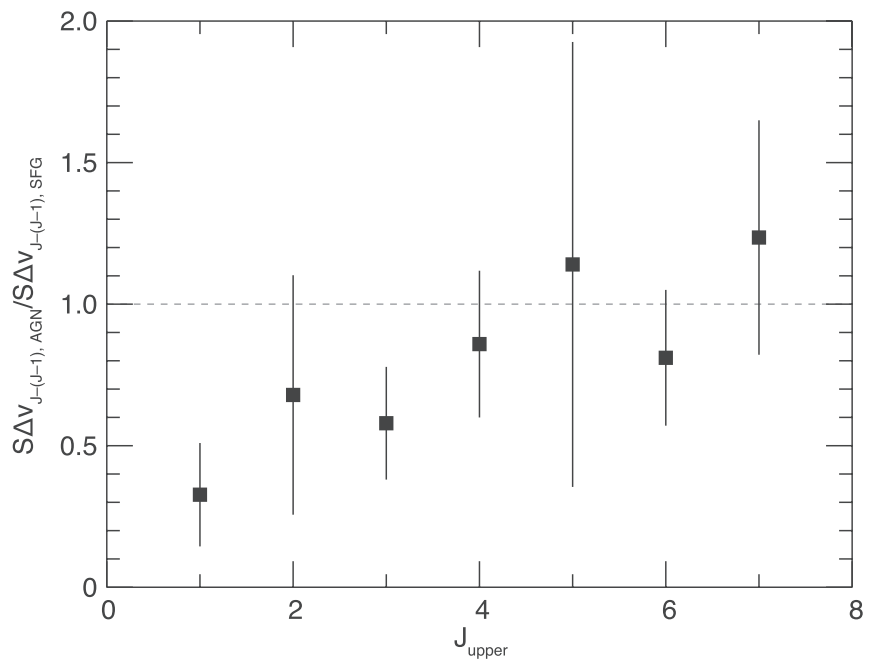

Figure 9. Median CO line fluxes for the galaxies with mid-IR AGN fractions $\geqslant 50 \%$ in ratio to the median $\mathrm{CO}$ line fluxes for the galaxies with mid-IR AGN fractions $<50 \%$. For clarity we show a gray dashed line for equal line fluxes between the two populations.

samples, where line fluxes have been scaled linearly by the ratio of their individual IR luminosity to the median IR luminosity of the full sample (Figure 9). While there are still substantial uncertainties on the population ratios for each line, the observed trend with $J$ is statistically significant; performing a Spearman's rank correlation test yields a $1.4 \%$ chance of getting an observed correlation at least this strong with a null hypothesis of no correlation. However, this correlation is not robust. When removing either of the extrema from the correlation test (either the $\mathrm{CO}(1-0)$ population ratio or the $\mathrm{CO}(7-6)$ population ratio), the probability increases to $7.6 \%$, which is sufficiently large that we likely cannot rule out the null hypothesis of no correlation. In addition, if we Monte Carlo over the uncertainties (since the Spearman's rank test does not factor those in), only $27 \%$ of iterations yield $p<0.05$ of getting an observed correlation at least this strong. Therefore we must conclude that our data do not show a significant difference between the $\mathrm{CO}$ excitation of SF-dominated and AGN-dominated galaxies as defined by $f(\mathrm{AGN})_{\mathrm{MIR}}=0.5$.

Ultimately, only two lines have $>5$ detections in both the AGN-dominated and SF-dominated samples: the $\mathrm{CO}(1-0)$ line with eight detections below and above $f(\mathrm{AGN})_{\mathrm{MIR}}=0.6$, and the $\mathrm{CO}(3-2)$ line with 15 and 18 detections below and above $f(\mathrm{AGN})_{\mathrm{MIR}}=0.6$ respectively (Table 4$)$. We find that the ratio of $\mathrm{CO}(1-0)$ fluxes for the AGN-dominated to SF-dominated galaxies is $0.33 \pm 0.18$, and the ratio for the $\mathrm{CO}(3-2)$ line is $0.58 \pm 0.20$. At face value, these low- $J$ ratios seem to indicate that for the same IR luminosity, $z>1$ galaxies with SFdominated mid-IR fluxes have more gas than $z>1$ galaxies with AGN-dominated mid-IR fluxes. Such systematically lower gas masses in high- $z$ AGNs might be expected if AGNs play a role in quenching periods of rapid star formation or if they are temporally correlated with the transition between more bursty and more quiescent phases of galaxy evolution. However, this result would be at odds with the observed lack of difference in SFEs found in Section 4.1. Due to the small sample size, more data are needed before drawing strong conclusions.

This observed difference may be artificially produced by our method of linearly scaling $L_{\mathrm{CO}}^{\prime}$ using the ratio of the total $L_{\mathrm{IR}}$ (including both the contributions from star formation and any 
AGNs) to some fiducial value. ${ }^{8}$ If the correlation between $L_{\mathrm{IR}}$ and $L_{\mathrm{CO}}^{\prime}$ (the integrated Schmidt-Kennicutt relation) is superlinear (or sub-linear in the case of Figure 5 where the axes are swapped), and $L_{\mathrm{IR}}$ of the AGN-dominated galaxies are $~ 0.5-1$ dex brighter than the SF-dominated galaxies on average, then in the process of linearly scaling $L_{\mathrm{CO}}^{\prime}$ we effectively over-scale the gas emission of the AGN-dominated sources and correspondingly under-scale that for the SF-dominated sources. These scalings would then compound to produce the relatively lower ratios of AGN-to-SF gas masses. This effect of the scaling may come into play in the average SLEDs produced in Section 3.4, since AGN-dominated and SF-dominated galaxies are averaged together. If the total $L_{\mathrm{IR}}$ distributions differ systematically with galaxy class (or some other parameter of interest) that has systematically differing $\mathrm{CO}$ excitation, and the $L_{\mathrm{IR}}-L_{\mathrm{CO}}^{\prime}$ is super-linear, then the net effect would be a bias toward the excitation characteristics of the lower-luminosity population. If the index of the Schmidt-Kennicutt correlation becomes shallower for higher- $J \mathrm{CO}$ gas tracers (e.g., Greve et al. 2014 and references therein; see Liu et al. 2015; Kamenetzky et al. 2016), then the applied scaling becomes systematically more appropriate for the higher- $J$ CO lines, and thus could also produce the observed correlation in Figure 9. In order to correct for such $L_{\mathrm{IR}}$-dependent effects, either we would need new observations of an $L_{\mathrm{IR}}$-matched sample with mid-IRdetermined AGN fractions, or we would need to find some other parameter that linearly scales with gas mass to use when calculating the median CO luminosities. However, such scaling effects produced by the different populations' $L_{\mathrm{IR}}$ distributions are removed by considering the higher- $J$ lines in ratio to the ground state (as for the CO SLED plots in Figure 8).

While high-J CO emission from IR-bright AGNs is welldocumented (e.g., Downes et al. 1999; Bertoldi et al. 2003; Walter et al. 2003; Weiss et al. 2007; Gallerani et al. 2014; Tuan-Anh et al. 2017), we do not find conclusive evidence for systematically higher-excitation CO SLEDs in this AGNdominated $f(\mathrm{AGN})_{\mathrm{MIR}}$ sample. In spatially resolved studies of galaxies, it is well known that regions with different heating sources (and thus cloud physical conditions; i.e., temperatures and densities) will produce different $\mathrm{CO}$ line ratios-cold molecular clouds with moderate star formation rates in the peripheries of disk galaxies will produce sub-thermal emission at even the lowest- $J$ CO lines (e.g., $r_{3,1}=0.4$ for the outer disk of the Milky Way; Fixsen et al. 1999), while dense starbursts' clouds or the central regions near AGNs can produce nearly thermalized emission to much higher $J$ (e.g., $r_{3,1}$ variations in the Antennae or near the central AGN of NGC 1068; Zhu et al. 2003; Spinoglio et al. 2012; Viti et al. 2014). Therefore, depending on the relative balance of these energy sources in each galaxy, we expect to measure different global line ratios (e.g., Harris et al. 2010; Narayanan \& Krumholz 2014). It follows that if the warm dust continuum emission from the AGN is dominating $L_{\mathrm{IR}}$, and dust and molecular gas is commingled, then we might expect to see higher excitation in such systems' CO SLEDs. Given the limitations of our current sample, we do not see evidence of this effect when comparing high- $z$ SFGs and IR AGNs as defined by $f(\mathrm{AGN})_{\mathrm{MIR}}$. Therefore, barring access to an $L_{\mathrm{IR}}$-matched sample with MIR-determined AGN fractions (which will become possible in the era of the James Webb Space Telescope (JWST)),

\footnotetext{
Scaling by $L_{\mathrm{IR}}^{\mathrm{SF}}$ does not change this result.
}

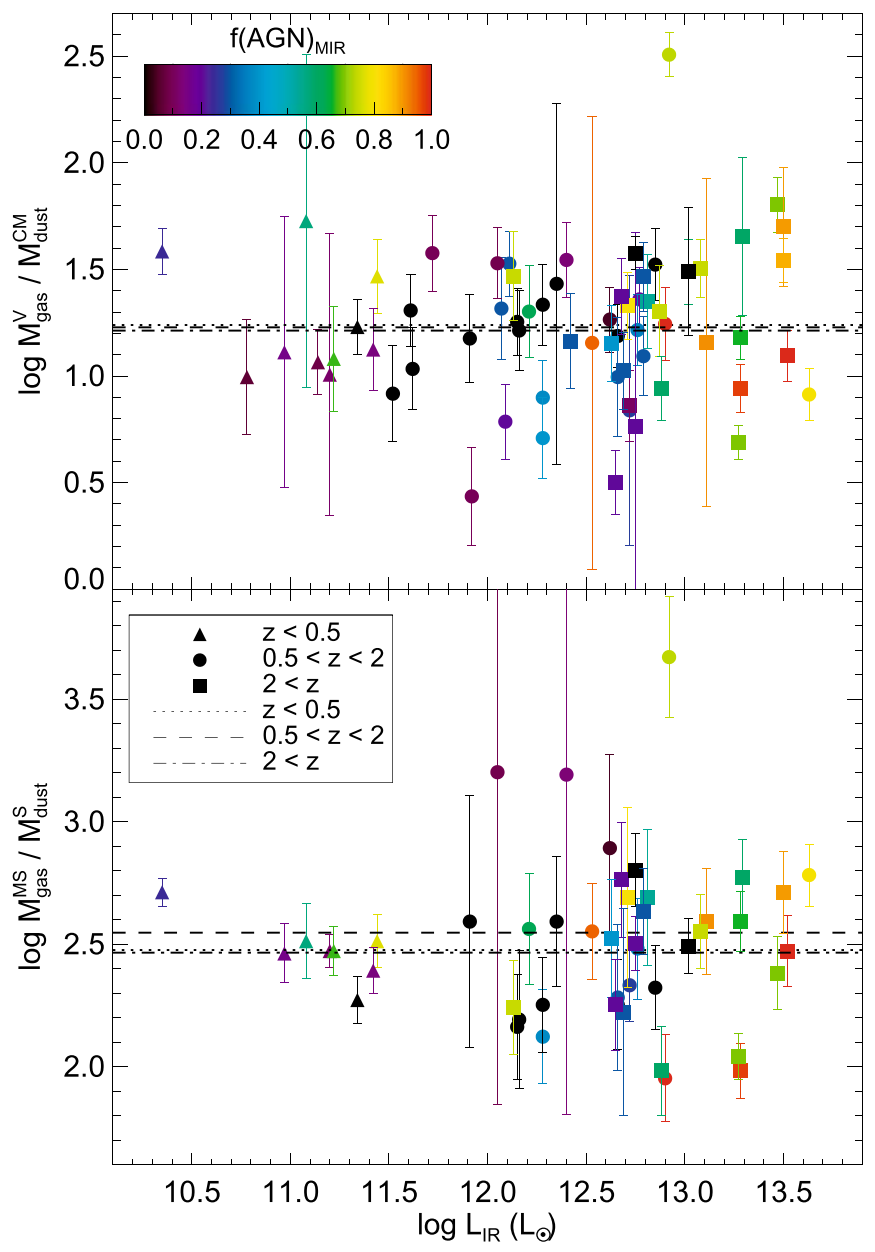

Figure 10. Gas to dust ratio (GDR) calculated with two different $M_{\text {gas }}$ and $M_{\text {dust }}$. Regardless of the methodology, GDR is consistent across redshift, $L_{\mathrm{IR}}$, and AGN presence. Top: $M_{\text {gas }}^{\mathrm{V}}$ is calculated with a variable $\alpha_{\mathrm{CO}}$ that depends on metallicity and ISM surface density. $M_{\text {dust }}^{\mathrm{CM}}$ is calculated by fitting far-IR observations with a dust radiative transfer model. We calculate the mean GDR values for three redshift bins: $z<0.5$ (dotted line), $0.5 \geqslant z<2$ (dashed line), $2 \geqslant z$ (dotted-dashed line). The mean values are all lower than expected ( 20). Bottom: GDR when $\alpha_{\mathrm{CO}}=4.6$ is used. $M_{\text {dust }}^{S}$ is calculated from submillimeter flux assuming $T_{C}=25 \mathrm{~K}$. The mean GDR for all three redshift bins is remarkably consistent: $\sim 300$.

increasing the number of multi-line $\mathrm{CO}$ detections (particularly at higher $J$ ) for archival Spitzer sources is likely the best way to improve statistics for the immediate future.

\subsection{Evolving Gas-to-dust Ratio}

Due to the relatively modest time required for continuum observations as compared with gas emission lines submillimeter emission has recently been promoted as a gas mass tracer (e.g., Groves et al. 2015; Scoville et al. 2016). Calibrators for converting dust continuum emission into a gas mass rely on the assumption that the gas-to-dust mass ratio (GDR) is constant in solar metallicity galaxies out to $z \sim 4$. We can test this assumption, since we have independently measured dust and gas masses in a heterogeneous sample of galaxies out to $z \approx 4$, and we can look for changes in the GDR with increasing $f(\mathrm{AGN})_{\mathrm{MIR}}$.

Figure 10 shows the GDR for our sample as a function of $L_{\mathrm{IR}}$. In the top panel, we use a varying $\alpha_{\mathrm{CO}}$ (Narayanan et al. 2012), and in the bottom panel, we use a constant 
$\alpha_{\mathrm{CO}}=4.6 M_{\odot}\left(\mathrm{K} \mathrm{km} \mathrm{s}^{-1} \mathrm{pc}^{2}\right)^{-1}$ for all galaxies, as described in Section 3.4.2. We determine the geometric mean of the GDR in three redshift bins and find it to be remarkably consistent; for a varying (constant) $\mathrm{CO}$-to- $\mathrm{H}_{2}$ conversion factor, the GDR is $17 \pm 3(300 \pm 28)$ for $z<0.5,17 \pm 3(353 \pm 81)$ for $0.5 \leqslant z<2$, and $16 \pm 3(292 \pm 34)$ for $z \geqslant 2$. We find no trend in GDR with $L_{\mathrm{IR}}$, and no difference in GDR between the sources above and below $f(\mathrm{AGN})_{\mathrm{MIR}}=0.5$. The exact GDR depends strongly on the assumed $\alpha_{\mathrm{CO}}$. It is difficult to know what the expected GDR at $z \sim 1-3$ is, since it hinges so sensitively on $\alpha_{\mathrm{CO}}$ and converting higher $\mathrm{CO}$ transitions to the ground state. In a sample of four solar metallicity galaxies at $z \sim 1.4$, Seko et al. (2016) use the $\mathrm{CO}(2-1)$ transition and $\alpha_{\mathrm{CO}}=4.36$ and calculate GDRs in the range 220-1450, in agreement with our measurements. Saintonge et al. (2013) measured the GDR in 17 lensed galaxies at $z=1.4-3.1$. They observed $\mathrm{CO}(3-2)$ and use a variable $\alpha_{\mathrm{CO}}$ based on metallicity. They derive GDRs in the range 100-700 and demonstrate that these values are $1.7 \times$ greater than for local galaxies. For local galaxies, Leroy et al. (2011) present a relation to derive GDR from metallicity. Based on the Leroy et al. relation, the expected average GDR of our galaxies is 50, closer to the value we derived following the Narayanan et al. (2012) method. The expected GDR, based on literature values for solar metallicity galaxies, is $100-200$. The use of $M_{\text {dust }}^{\mathrm{CM}}$ rather than $M_{\text {dust }}^{\mathrm{S}}$ would increase the GDR by a small amount (the most significant increase is for $0.5 \leqslant z<2$ where the GDR increases to $\sim 350$ ).

The GDRs calculated using the varying $\alpha_{\mathrm{CO}}$ are very low. These low GDRs are due to the low gas masses inferred from the lower $\mathrm{CO}-$ to- $\mathrm{H}_{2}$ conversion factors; these low conversion factors are either due to small radii used in calculating the $\mathrm{CO}$ surface brightness $\left(\Sigma_{\mathrm{CO}}\right)$ or overestimated metallicities. Large $\Sigma_{\mathrm{CO}}$ (and thus low GDRs) could occur if dust and gas is not cospatial and the dust emission in high- $z$ SMGs is particularly compact (e.g., Hodge et al. 2016). If we assume that all galaxies have solar metallicity, then the average radius of the $\mathrm{CO}$ emission would need to be a factor of ten larger than the typical dust emission to produce GDR $\sim 100-200$ in all galaxies. Indeed, if we assume that all galaxies have solar metallicity, then there would be a consistent increase in GDR with redshift, so that by $z \geqslant 2, \mathrm{GDR}=34$. Alternatively, if the low GDR is due to an overestimate of the metallicities, we would need to drop metallicities to $Z<8.0$ in order to produce GDRs of $\sim 100$. Such a drop in metallicity would imply a significant evolution in the relationship between $M_{\text {dust }}$ and metallicity in massive galaxies as a function of redshift.

Assuming that any concerns with the CO-to- $\mathrm{H}_{2}$ conversion factor are satisfied, our values of the GDR indicate that the varying assumptions used for calculating $M_{\text {gas }}$ and $M_{\text {dust }}$ likely require adjustment for galaxies beyond the local universe. Additional concerns about $\alpha_{\mathrm{CO}}$ arise from estimating the gas phases at high redshift, particularly since more hydrogen may be in the molecular phase rather than the atomic phase compared with the local universe (Bertemes et al. 2018; Janowiecki et al. 2018). Regardless of method or assumptions, the GDR is consistent for all $f(\mathrm{AGN})_{\mathrm{MIR}}$ across the range of redshifts probed here. However, for individual objects, the ratios can vary significantly. Using the dust emission to estimate gas emission is likely only valid on average across large samples, rather than for individual objects.

\section{Conclusions and Future Directions}

We have compiled a heterogeneous sample of 67 galaxies from the literature that have $\mathrm{CO}$ line measurements and IR spectroscopy/photometry from $z=0-4$. We have diagnosed the presence of an IR AGN using spectral decomposition or IR color selection based on techniques calibrated for galaxies at $z \sim 1-3$, and measured $L_{\mathrm{IR}}, M_{\text {dust }}$, and $L_{\mathrm{CO}}^{\prime}$ consistently for all sources. Our findings are as follows.

1. SFE is not strongly correlated with AGN emission. Strong AGNs are observed to have both high and low SFEs, indicating that the presence of an AGN itself does not indicate a galaxy is beginning to quench. Any expected correlation may necessarily be murky due to AGN luminosity flickering on much shorter timescales than galaxy evolution.

2. It is important to fully sample the IR SED, use appropriate templates, and take AGN heating effects into account when calculating $L_{\mathrm{IR}}$. If this is not done, derived SFRs will be too high and SFE will wrongly appear to be enhanced in IR AGNs.

3. GDRs do not appear to evolve with redshift for this heterogeneous sample, although the scatter is large. We find consistent GDRs regardless of redshift, luminosity, or AGN strength. However, for individual objects, the exact GDR can vary significantly.

4. We do not find a statistically significant difference in the $\mathrm{CO}$ excitation for individual line ratios for galaxies above and below $f(\mathrm{AGN})_{\mathrm{MIR}}=0.5$. While it appears that the median CO SLED of sources with $f(\mathrm{AGN})_{\mathrm{MIR}} \geqslant 0.5$ is at systematically higher excitation than median CO SLED of galaxies with $f(\mathrm{AGN})_{\mathrm{MIR}}<0.5$ for all rotational transitions, this result is not robust statistically.

Many of our conclusions about the molecular gas content and the effects of AGN heating is limited by small-number statistics and the lack of matched luminosity samples. A systematic CO SLED survey of galaxies with known IR AGN content (i.e., measured $f(\mathrm{AGN})_{\mathrm{MIR}}$ ) is critical for disentangling evidence of AGN feedback affecting star-forming molecular gas via the gas physical conditions. Since the problem of high SFRs that require some limiting mechanism to prevent the over-production of massive galaxies today is particularly acute among dusty SFGs, studies of AGN feedback are more likely to be successful when focused on high- $z$, IR-bright galaxies such as SMGs. Since comparing average gas properties requires scaling out the dominant effect of brighter/moremassive galaxies being brighter across the electromagnetic spectrum (to first order), accurate normalization is similarly critical. Firmer conclusions could be reached with either (a) more observations of the $\mathrm{CO}(1-0)$ line to normalize the excitation, (b) more observations of the $J \geqslant 5 \mathrm{CO}$ lines where the number of detections for $f(\mathrm{AGN})_{\mathrm{MIR}}$ samples are lowest, or (c) developing matched IR luminosity samples among IR AGNs and SFGs. Until we have the mid-IR spectroscopic/ photometric capabilities of the JWST that enable the selection of matched $L_{\mathrm{IR}}$ samples of high- $z$ galaxies, we are restricted to analyzing galaxies with archival Spitzer data.

A.K. thanks Sukanya Chakrabarti and Desika Narayanan for helpful conversations. A.K. gratefully acknowledges support from the YCAA Prize Postdoctoral Fellowship. 


\section{ORCID iDs}

Chelsea Sharon (1) https://orcid.org/0000-0002-6250-5608 Alexandra Pope (1) https://orcid.org/0000-0001-8592-2706

\section{References}

Ao, Y., Weiß, A., Downes, D., et al. 2008, A\&A, 491, 747

Aravena, M., Hodge, J. A., Wagg, J., et al. 2014, MNRAS, 442, 558

Bertemes, C., Wuyts, S., Lutz, D., et al. 2018, MNRAS, 478, 1442

Bertoldi, F., Cox, P., Neri, R., et al. 2003, A\&A, 409, L47

Blain, A. W. 1999, MNRAS, 304, 669

Bolatto, A. D., Wolfire, M., \& Leroy, A. K. 2013, ARA\&A, 51, 207

Bothwell, M. S., Smail, I., Chapman, S. C., et al. 2013, MNRAS, 429, 3047

Bouché, N., Dekel, A., Genzel, R., et al. 2010, ApJ, 718, 1001

Brinchmann, J., Charlot, S., White, S. D. M., et al. 2004, MNRAS, 351, 1151

Caplar, N., Lilly, S. J., \& Trakhtenbrot, B. 2018, ApJ, 867, 148

Carilli, C. L., \& Walter, F. 2013, ARA\&A, 51, 105

Casey, C. M., Narayanan, D., \& Cooray, A. 2014, PhR, 541, 45

Chakrabarti, S., \& McKee, C. F. 2005, ApJ, 631, 792

Chakrabarti, S., \& McKee, C. F. 2008, ApJ, 683, 693

Chang, Y.-Y., Ferraro, N., Wang, W.-H., et al. 2018, ApJ, 865, 103

Chary, R., \& Elbaz, D. 2001, ApJ, 556, 562

Cicone, C., Maiolino, R., Sturm, E., et al. 2014, A\&A, 562, A21

Croton, D. J., Springel, V., White, S. D. M., et al. 2006, MNRAS, 365, 11

da Cunha, E., Groves, B., Walter, F., et al. 2013, ApJ, 766, 13

Daddi, E., Dickinson, M., Morrison, G., et al. 2007, ApJ, 670, 156

Daddi, E., Elbaz, D., Walter, F., et al. 2010, ApJL, 714, L118

Dale, D. A., \& Helou, G. 2002, ApJ, 576, 159

Davé, R., Oppenheimer, B. D., \& Finlator, K. 2011, MNRAS, 415, 11

Delvecchio, I., Gruppioni, C., Pozzi, F., et al. 2014, MNRAS, 439, 2736

Downes, D., Neri, R., Wiklind, T., Wilner, D. J., \& Shaver, P. A. 1999, ApJL, 513, L1

Draine, B. T. 2003, ARA\&A, 41, 241

Draine, B. T., \& Li, A. 2007, ApJ, 657, 810

Elbaz, D., Daddi, E., Le Borgne, D., et al. 2007, A\&A, 468, 33

Elvis, M., Wilkes, B. J., McDowell, J. C., et al. 1994, ApJS, 95, 1

Ferrarese, L., \& Merritt, D. 2000, ApJL, 539, L9

Feruglio, C., Maiolino, R., Piconcelli, E., et al. 2010, A\&A, 518, L155

Fiore, F., Feruglio, C., Shankar, F., et al. 2017, A\&A, 601, A143

Fixsen, D. J., Bennett, C. L., \& Mather, J. C. 1999, ApJ, 526, 207

Frayer, D. T., Maddalena, R. J., Ivison, R. J., et al. 2018, ApJ, 860, 87

French, K. D., Yang, Y., Zabludoff, A., et al. 2015, ApJ, 801, 1

French, K. D., Zabludoff, A. I., Yoon, I., et al. 2018, ApJ, 861, 123

Gabor, J. M., Davé, R., Oppenheimer, B. D., \& Finlator, K. 2011, MNRAS, 417, 2676

Gallerani, S., Ferrara, A., Neri, R., \& Maiolino, R. 2014, MNRAS, 445, 2848

Gebhardt, K., Bender, R., Bower, G., et al. 2000, ApJL, 539, L13

Genzel, R., Tacconi, L. J., Gracia-Carpio, J., et al. 2010, MNRAS, 407, 2091

Glikman, E., Urrutia, T., Lacy, M., et al. 2012, ApJ, 757, 51

Goulding, A. D., Greene, J. E., Bezanson, R., et al. 2018, PASJ, 70, S37

Gowardhan, A., Spoon, H., Riechers, D. A., et al. 2018, ApJ, 859, 35

Greve, T. R., Leonidaki, I., Xilouris, E. M., et al. 2014, ApJ, 794, 142

Groves, B., Dopita, M., \& Sutherland, R. 2006, A\&A, 458, 405

Groves, B. A., Schinnerer, E., Leroy, A., et al. 2015, ApJ, 799, 96

Hailey-Dunsheath, S., Sturm, E., Fischer, J., et al. 2012, ApJ, 755, 57

Hainline, K. N., Hickox, R., Greene, J. E., Myers, A. D., \& Zakamska, N. L. 2013, ApJ, 774, 145

Hainline, K. N., Hickox, R. C., Chen, C.-T., et al. 2016, ApJ, 823, 42 Harris, A. I., Baker, A. J., Zonak, S. G., et al. 2010, ApJ, 723, 1130

Harrison, C. M., Alexander, D. M., Mullaney, J. R., et al. 2012, ApJ, 760, L15

Herrera-Camus, R., Tacconi, L., Genzel, R., et al. 2019, ApJ, 871, 37

Hezaveh, Y. D., Marrone, D. P., \& Holder, G. P. 2012, ApJ, 761, 20

Hickox, R. C., Mullaney, J. R., Alexander, D. M., et al. 2014, ApJ, 782, 9

Hodge, J. A., Riechers, D., Decarli, R., et al. 2015, ApJL, 798, L18

Hodge, J. A., Smail, I., Walter, F., et al. 2019, ApJ, 876, 130

Hodge, J. A., Swinbank, A. M., Simpson, J. M., et al. 2016, ApJ, 833, 103

Hopkins, P. F., Somerville, R. S., Hernquist, L., et al. 2006, ApJ, 652, 864

Hunt, Q., Bezanson, R., Greene, J. E., et al. 2018, ApJL, 860, L18

Ichikawa, K., \& Tazaki, R. 2017, ApJ, 844, 21

Ishibashi, W., \& Fabian, A. C. 2012, MNRAS, 427, 2998

Janowiecki, S., Cortese, L., Catinella, B., \& Goodwin, A. J. 2018, MNRAS, 476,1390

Kamenetzky, J., Rangwala, N., Glenn, J., Maloney, P. R., \& Conley, A. 2016, ApJ, 829, 93
Kartaltepe, J. S., Dickinson, M., Alexander, D. M., et al. 2012, ApJ, 757, 23 Kennicutt, R. C., Jr. 1989, ApJ, 344, 685

Kim, C.-G., Ostriker, E. C., \& Kim, W.-T. 2013, ApJ, 776, 1

Kirkpatrick, A., Alberts, S., Pope, A., et al. 2017, ApJ, 849, 111

Kirkpatrick, A., Pope, A., Alexander, D. M., et al. 2012, ApJ, 759, 139

Kirkpatrick, A., Pope, A., Aretxaga, I., et al. 2014, ApJ, 796, 135

Kirkpatrick, A., Pope, A., Sajina, A., et al. 2015, ApJ, 814, 9

Kocevski, D. D., Barro, G., Faber, S. M., et al. 2017, ApJ, 846, 112

Kormendy, J., \& Ho, L. C. 2013, ARA\&A, 51, 511

Leroy, A. K., Bolatto, A., Gordon, K., et al. 2011, ApJ, 737, 12

Lilly, S. J., Carollo, C. M., Pipino, A., Renzini, A., \& Peng, Y. 2013, ApJ, 772,119

Liu, D., Gao, Y., Isaak, K., et al. 2015, ApJL, 810, L14

Mahoro, A., Pović, M., \& Nkundabakura, P. 2017, MNRAS, 471, 3226

Misselt, K. A., Gordon, K. D., Clayton, G. C., \& Wolff, M. J. 2001, ApJ, 551,277

Mullaney, J. R., Alexander, D. M., Goulding, A. D., \& Hickox, R. C. 2011 , MNRAS, 414, 1082

Murphy, E. J., Condon, J. J., Schinnerer, E., et al. 2011, ApJ, 737, 67

Narayanan, D., \& Krumholz, M. R. 2014, MNRAS, 442, 1411

Narayanan, D., Krumholz, M. R., Ostriker, E. C., \& Hernquist, L. 2012, MNRAS, 421, 3127

Narayanan, D., Turk, M., Feldmann, R., et al. 2015, Natur, 525, 496

Noeske, K. G., Weiner, B. J., Faber, S. M., et al. 2007, ApJL, 660, L43

Page, M. J., Symeonidis, M., Vieira, J. D., et al. 2012, Natur, 485, 213

Perna, M., Sargent, M. T., Brusa, M., et al. 2018, A\&A, 619, A90

Pontzen, A., Tremmel, M., Roth, N., et al. 2017, MNRAS, 465, 547

Pope, A., Chary, R.-R., Alexander, D. M., et al. 2008, ApJ, 675, 1171

Pope, A., Wagg, J., Frayer, D., et al. 2013, ApJ, 772, 92

Rémy-Ruyer, A., Madden, S. C., Galliano, F., et al. 2015, A\&A, 582, A121

Revalski, M., Dashtamirova, D., Crenshaw, D. M., et al. 2018, ApJ, 867, 88

Ricci, C., Assef, R. J., Stern, D., et al. 2017, ApJ, 835, 105

Riechers, D. A., Carilli, C. L., Maddalena, R. J., et al. 2011a, ApJL, 739, L32

Riechers, D. A., Carilli, L. C., Walter, F., et al. 2011b, ApJL, 733, L11

Rosenberg, M. J. F., van der Werf, P. P., Aalto, S., et al. 2015, ApJ, 801, 72 Saintonge, A., Lutz, D., Genzel, R., et al. 2013, ApJ, 778, 2

Sajina, A., Yan, L., Fadda, D., Dasyra, K., \& Huynh, M. 2012, ApJ, 757, 13 Sanders, D. B., \& Mirabel, I. F. 1996, ARA\&A, 34, 749

Sargent, M. T., Béthermin, M., Daddi, E., \& Elbaz, D. 2012, ApJL, 747, L31

Sargent, M. T., Daddi, E., Béthermin, M., et al. 2014, ApJ, 793, 19

Schmidt, M. 1959, ApJ, 129, 243

Scoville, N., Sheth, K., Aussel, H., et al. 2016, ApJ, 820, 83

Seko, A., Ohta, K., Yabe, K., et al. 2016, PASJ, 68, 62

Serjeant, S. 2012, MNRAS, 424, 2429

Sharon, C. E., Riechers, D. A., Hodge, J., et al. 2016, ApJ, 827, 18

Shetty, R., \& Ostriker, E. C. 2012, ApJ, 754, 2

Silk, J. 2013, ApJ, 772, 112

Solomon, P. M., \& Barrett, J. W. 1991, in IAU Symp. 146, Dynamics of Galaxies and Their Molecular Cloud Distributions, ed. F. Combes \& F. Casoli (Dordrecht: Kluwer), 235

Solomon, P. M., Downes, D., Radford, S. J. E., \& Barrett, J. W. 1997, ApJ, 478,144

Somerville, R. S., Hopkins, P. F., Cox, T. J., Robertson, B. E., \& Hernquist, L. 2008, MNRAS, 391, 481

Spilker, J. S., Aravena, M., Béthermin, M., et al. 2018, Sci, 361, 1016

Spinoglio, L., Pereira-Santaella, M., Busquet, G., et al. 2012, ApJ, 758, 108

Stacey, G. J., Hailey-Dunsheath, S., Ferkinhoff, C., et al. 2010, ApJ, 724, 957

Stanley, F., Alexander, D. M., Harrison, C. M., et al. 2017, MNRAS, 472, 2221

Suess, K. A., Bezanson, R., Spilker, J. S., et al. 2017, ApJL, 846, L14

Symeonidis, M., Giblin, B. M., Page, M. J., et al. 2016, MNRAS, 459, 257

Tacconi, L. J., Neri, R., Genzel, R., et al. 2013, ApJ, 768, 74

Tadaki, K.-i., Kodama, T., Nelson, E. J., et al. 2017, ApJL, 841, L25

Tuan-Anh, P., Hoai, D. T., Nhung, P. T., et al. 2017, MNRAS, 467, 3513

Viti, S., García-Burillo, S., Fuente, A., et al. 2014, A\&A, 570, A28

Walter, F., Bertoldi, F., Carilli, C., et al. 2003, Natur, 424, 406

Weiß, A., Downes, D., Neri, R., et al. 2007, A\&A, 467, 955

Weingartner, J. C., \& Draine, B. T. 2001, ApJ, 548, 296

Weiss, A., Downes, D., Walter, F., \& Henkel, C. 2007, in ASP Conf. Ser. 375,

From Z-Machines to ALMA: (Sub)Millimeter Spectroscopy of Galaxies, ed. A. J. Baker et al. (San Francisco, CA: ASP), 25

Witt, A. N., \& Gordon, K. D. 2000, ApJ, 528, 799

Xu, L., Rieke, G. H., Egami, E., et al. 2015, ApJ, 808, 159

Yang, C., Omont, A., Beelen, A., et al. 2017, A\&A, 608, A144

Zhu, M., Seaquist, E. R., \& Kuno, N. 2003, ApJ, 588, 243 